\title{
SUPERCRITICAL FLUID THERMODYNAMICS FOR COAL PROCESSING
}

Final Report for the Period September 15, 1988-September 14, 1991

\author{
By \\ Frank van Swol \\ Charles A. Eckert
}

September 15, 1988

Work Performed Under Contract No. DE-FG22-88PC88922

For

U.S. Department of Energy

Pittsburgh Energy Technology Center

Pittsburgh, Pennsylvania

By

Georgia Institute of Technology

Atlanta, Georgia 


\section{CONTENTS}

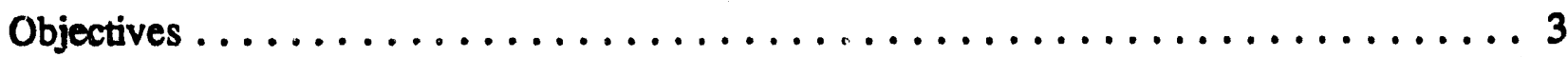

Phase Behavior $\ldots \ldots \ldots \ldots \ldots \ldots \ldots \ldots \ldots \ldots \ldots \ldots \ldots \ldots \ldots \ldots \ldots \ldots \ldots$

High Temperature Solubility Studies $\ldots \ldots \ldots \ldots \ldots \ldots \ldots \ldots$

Ultraviolet Absorption Spectroscopy for Measuring Solubility . . . . . . . . . 4

Chromatographic Technique to Study Solubilities and Cosolvent Effects ...... 5

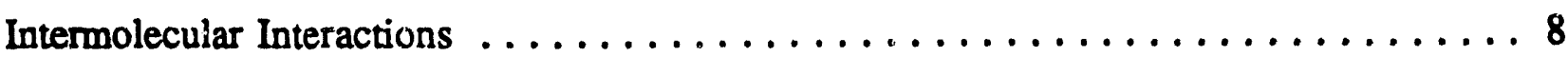

Solute/Solvent Interactions $\ldots \ldots \ldots \ldots \ldots \ldots \ldots \ldots \ldots \ldots \ldots \ldots \ldots$

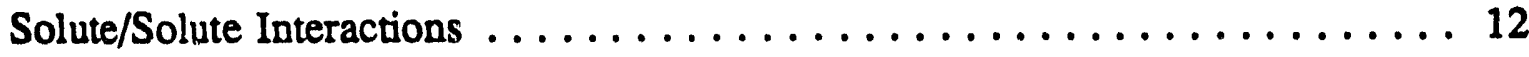

Intermolecular Interactions in Cosolvent Solutions . . . . . . . . . . . 13

Chemical-Physical Modelling of Molecular Charisma and Cosolvent Effects . . . . . 17

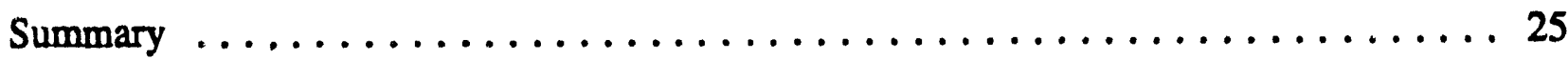

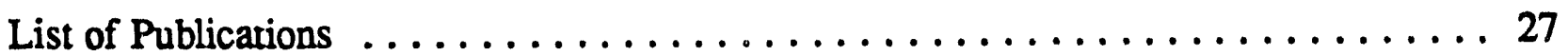

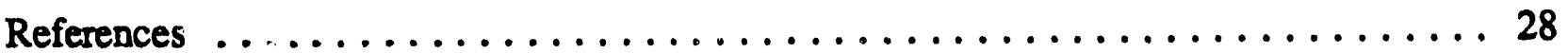




\begin{abstract}
Because of their unusual solvating and mass transfer properties, supercritical fluids show potential for a variety of coal processing applications. We have established a database of coal model compound equilibria; to add to this database, we have developed and are testing techniques of measuring cosolvent effects nn solubility rapidly. In addition, we have used fluorescence spectroscopy to study the nature of cosolvent effects on a molecular level. The solubility and spectroscopic measurements are being used in the development of an equation of state that includes both physical and chemical interactions; we are currently testing the equation. The equation of state will be used to predict solubility behavior so systems can be designed for the processing of coal with supercritical fluids.
\end{abstract}




\section{OBJECTIVES}

The main objective of this research is to develop an equation of state that can be used to predict solubilities and tailor supercritical fluid solvents for the extraction and processing of coal. To meet this objective we have implemented a two-sided approach. First, we expanded the database of model coal compound solubilities in higher temperature fluids, polar fluids, and tluid mixtures systems. Second, the unique solute/solute, solute/cosolvent and solute/solvent intermolecular interactions in supercritical fluid solutions were investigated using spectroscopic techniques. These results increased our understanding of the molecular phenomena that affect solubility in supercritical fluids and were significant in the development of an equation of state (EOS) that accurately reflects the true molecular makeup of the solution.

Supercritical fluids (SCF) show potential for coal processing because they possess some unique properties. A SCF is quite dense, which gives a large capacity for solutes; it has high diffusivity and low viscosity, which makes it an ideal medium for efficient mass transfer, and the fluid is highly compressible, which yields large solubility changes with small changes in temperature and pressure. This suggests extraordinary selectivity possibilities.

Many of the SCF investigations to date have been with relatively mild, low-temperature fluids like carbon dioxide. However, any process for coal conversion will require more aggressive, higher temperature and more polar SCF solvents like ammonia, methanol, and butane. In addition, we have found that the addition of a small percentage of a different component that specifically interacts with the solute (a "cosolvent") can dramatically increase the solubility of heavy organic solutes. Therefore, we have measured the solubilities of model coal compounds in these novel fluids and fluid mixtures. In addition to a high-temperature apparatus capable of measuring solubilities in SCF solutions with higher critical points, we have also used two novel techniques to measure rapidly solubilities in pure SCF's and cosolvent mixtures.

Finally, in order to develop an equation of state that can be used to predict as well as correlate solubility data, an understanding of the actual intermolecular interactions is required. To probe those interactions we have developed a high pressure fluorescence spectroscopy apparatus. From the spectra, information can be derived about the strength of both solute/solvent and solute/solute forces and how they change with proximity to the critical point. Both solubility and spectroscopic data are vital to the development of an equation of state model that accounts for both the physical and chemical forces in SCF solutions. 


\section{PHASE BEHAVIOR}

We have compiled an extensive database of coal model compound solubilities. The dearth of information on the solubilities of these compounds in higher temperature supercritical fluids and in cosolvent solutions limits the application of SCF solutions to coal processing. In addition, further equilibria data were necessary to the development of an accurate equation of state which could be used to tailor SCF solutions to specific coal processes.

\section{High Temperature Solubility Studies}

In general, $\mathrm{CO}_{2}$ has been the primary solvent used in SCF solubility studies. Although it is inexpensive, non-toxic, and has a low critical temperature, it is not the optimum solvent in many cases due to its low polarizability. Solubilities in SCF's with higher critical temperatures are enhanced by the higher solute vapor pressure as well as a generally increased solvent polarizability compared to $\mathrm{CO}_{2}$. Applications of SCF technology to coal processing may require these stronger solvents and higher temperatures. Solubilities determined in SCF's at higher temperatures are alsi important to the development of an equation of state that accurately represents SCF solutions.

The solubilities of 5,6 dimethylbenzimidazole, anthraquinone, and 9,10 phenanthrenequinone were measured in SCF butane at $155^{\circ} \mathrm{C}$ to $185^{\circ} \mathrm{C}$ at pressures between 42 and 77 bar. The critical point of butane is $152.1^{\circ} \mathrm{C}$ and 37.9 bar. These data are shown in Figures 1, 2, and 3 and exhibit the characteristic "crossover region", below which the solubility decreases with increasing temperatures.

\section{Ultraviolet Absorption Spectroscopy for Measuring Solubility}

A modified double-beam high-pressure UV absorption apparatus was used to determine solubilities in SCF fluid solutions (Figure 4). This novel technique is applicable to solids with solubilities in the range of commercial absorptions spectrometers, or mole fractions between $10^{-6}$ and $10^{-2}$. Many of the solids of interest are aromatic in nature and possess relatively strong UV-vis absorption characteristics. Beer's law, which is applicable for low concentrations, states that the absorption of light is proportional to the concentration of the sample. By using a double beam experiment, the complication of correcting the spectra for variations in solvent refractive index, which changes dramatically through the critical region, was avoided. Through a calibration curve of absorbance vs. concentration for solutions below the saturation limit, an absorbance reading of a saturated solution yields the concentration of the solution and hence the solubility.

The test the apparatus, we measured the solubility of anthracene in supercritical $\mathrm{CO}_{2}$ at $35^{\circ} \mathrm{C}$. Since literature data was available (Dobbs and Johnston, 1986; Kosal and Holder, 1987), the region of calibration was known in advance. The resulting solubility measurements 
are shown in Figure 5. Quite good agreement was obtained, confirming that the apparatus was functional. Solubility measurements were than made for PRODAN (6-propionyl-2dimethylaminonaphthalene), a spectroscopic probe molecule. We determined in our study of carbazole and 7-azaindole in several SCF solvents that this apparatus is not suited to the solubility measurement of highly soluble compounds with high extinction coefficients. These compounds exceed the absorbance range of the apparatus before the solubility limit is reached.

\section{Chromatographic Technique to Study Solubility and Cosolvent Effects in SCF Solutions}

Supercritical fluids (SCF's) have many special properties that make them ideal candidates for separations; however, selectivities depend primarily on volatilities (Dobbs and Johnston, 1987). One promising approach for manipulating selectivities is the addition of a small amount of cosolvent to the SCF. For example, the solubility of salicylic acid increases by more than an order of magnitude in $\mathrm{CO}_{2}$ with $3.5 \mathrm{~mol} \%$ methanol relative to pure $\mathrm{CO}_{2}$ (Foster, 1990). Nevertheless, current applications of cosolvent separations are both sparse and empirical, largely because of a lack of experimental data and accurate models.

One reason that data are scarce is that conventional techniques to measure solubilities in SCFs are time-consuming and require large amounts of pure solute. The chromatographic technique that we have developed to measure solubilities and the effects of cosolvents in SCF solutions is attractive because chromatographic measurements are rapid, require little solute, and inherently separate impurities from the sointe.

Several previous researchers have used supercritical fluid chromatography (SFC) to make thermodynamic measurements in pure SCFs. Solubility isotherms for a solute in pure solvents can be measured rapidly using SFC when an independently determined solubility point for the solute is available at the same temperature (Smith et al., 1987; Bartle et al., 1990a, 1990b). Brown et al. (1987) measured stationary phase Henry's constants for several solutes and correlated them with solute properties. This technique makes possible the measurement of solubilities from retention data where no additional solubility data exist. Shim and Johnston (1991) measured the distribution coefficients for naphthalene and phenanthrene between SCF $\mathrm{CO}_{2}$ and a bonded octadecane liquid phase in addition to solute partial molar volumes and enthalpies in the fluid phase.

The retention time of a solute depends upon how it is distributed between the mobile phase (in this case, a SCF) and the stationary phase, and is characterized by $k_{i}$, the dimensionless capacity factor

$$
k_{i}=\frac{t_{i}-t_{0}}{t_{0}}
$$

where $t_{i}$ is the retention time of solute $i$ and $t_{0}$ is the retention time of a solute that does not interact with the stationary phase. Assuming that equilibrium partitioning of the solute between the mobile and stationary phases is the sole mechanism of solute retention, the capacity factor is related to the equilibrium distribution of the solute between the two phases by 


$$
k_{i}=\frac{x_{i}}{y_{i}} \frac{v^{F}}{v^{s}} \frac{V^{S}}{V^{F}}
$$

where $x_{i}$ and $y_{i}$ are the mole fractions of component $i$ in the stationary and fluid phases respectively, $v^{F}$ and $v^{S}$ are the molar volumes of the fluid and stationary phases, and $V^{F}$ and $W^{S}$ are the volumes of the fluid and stationary phases. The validity of this assumption is discussed later.

The fugacity of the solute in the fluid phase, $f_{i}$, is

$$
f_{i}^{F}=y_{i} \phi_{i}^{\infty} P
$$

where $\phi_{i}^{\infty}$ is the fugacity coefficient of solute $i$ at infinite dilution and $P$ is the pressure. Assuming Henry's law is valid in the stationary phase gives

$$
f_{i}^{S}=x_{i} H_{i}
$$

where $H_{i}$ is the Henry's constant. At equilibrium, the fugacities are equal; substituting equations (3) and (4) into (2) then gives

$$
\phi_{i}^{-*}=\frac{k_{l} H_{i}}{P v^{F}} \frac{V^{F} v^{S}}{V^{S}}
$$

The ratio $V^{F} v^{S} / V^{S}$ depends on the column and should not vary significantly with column conditions. To determine the fugacity of a solute along an isotherm at a series of pressures in the fluid phase then requires knowledge of Henry's constant at that temperature.

Besides equilibrium partitioning, other mechanisms of solute retention that must be considered include adsorption of the solute at the interface between the mobile and stationary phases and interaction with active sites on the stationary phase support. These are very difficult to quantify and are usually ignored when making thermodynamic measurements with chromatography. Here, we use a comparison technique that cancels out the effects of retention mechanisms other than bulk absorption.

In our work, we compare the retention of a solute at the same temperature and pressure in the same column, but with different fluids as the mobile phase. Taking the ratio of equation (5) for two different mobile phases (denoted by ' and ") gives

$$
\frac{\phi_{i}^{\prime \prime}}{\phi_{i}^{\prime \prime}}=\frac{k_{i}^{\prime}}{k_{i}^{\prime \prime}} \frac{H_{i}^{\prime}}{H_{i}{ }^{\prime \prime}} \frac{v^{F \prime}}{v^{F^{\prime}}} \frac{V^{F^{\prime}} v^{S^{\prime}} V^{S^{\prime \prime}}}{V^{F^{\prime \prime}} v^{S^{\prime \prime}} V^{S^{\prime}}}
$$

Several simplifying assumptions may be made. First, because the volume of the mobile phase is mach greater than the volume of the stationary phase in a capillary column, swelling of the stationary phase has negligible effect on $V^{F}$, thus it can be considered constant. Second, the ratio $v^{S} / V^{S}$, or the total number of moles in the stationary phase, should not depend greatly upon the mobile phase. 
The third and key assumption is that Henry's constant for a solute is not altered by the changes in composition of the mobile phase. Several researchers have measured significant absorption of cosolvents into stationary phases (for example, Yonker and Smith, 1990; Strubinger et al., 1991). It has been proposed that the cosolvent covers up active sites on the column; this would cause the solute to be retained less thus decreasing the retention time (Levy and Ritchey, 1985). However, later studies have demonstrated that the increase in solvating power of the fluid upon addition of the cosolvent is primarily responsible for the decrease in retention implying that the stationary phase environment around the solute is unaffected by the cosolvent (Berger and Deye, 1990; Crow and Foley, 1991). Our work also seems to indicate that the mobile phase solvent strength is the primary factor.

After making these assumptions, equation (6) becomes

$$
\frac{\phi_{i}^{\alpha^{\prime \prime}}}{\phi_{l}^{{ }^{\prime \prime}}}=\frac{k_{t}{ }^{\prime \prime}}{k_{l}{ }^{\prime \prime}} \frac{v^{F^{\prime \prime}}}{v^{p^{\prime}}}
$$

The ratio of the molar volumes should be near unity and can be estimated from an equation of state, from experimental data, or from the $t_{0}$ and flow rate measurements.

At saturation

$$
\frac{y_{i}^{\operatorname{sen} \prime \prime}}{y_{i}^{\operatorname{sen} t^{\prime}}}=\frac{\phi_{i}^{\operatorname{sat}}}{\phi_{i}^{\operatorname{sen} \prime^{\prime \prime}}}
$$

If the solubilities of the solute in the SCFs are low (as is typically the case), it is reasonable to assume that the ratio of fugacities at the solubility limits is the same as that at infinite dilution. If so, then

$$
\Psi=\frac{y_{i}{ }^{\prime \prime}}{y_{i}{ }^{\prime}} \approx \frac{\phi_{i}^{-\prime}}{\phi_{i}{ }^{\prime \prime}}
$$

where $\Psi$ is the cosolvent effect; i.e., the relative increase in solubility at some $P$ and $T$ that comes about when the SCF is modified with cosolvent. To obtain the absolute solubility of the solute in the cosolvent solution using this technique, it is necessary to know the solute solubility in the pure SCF.

Infinite dilution fugacity coefficients are a more useful measure of solution thermodynamics than solubilities for theoretical techniques of studying solutions such as statistical mechanics or computer simulations. Solute-solute interactions are eliminated at infinite dilution, thus nonidealities under these conditions are due solely to solute-solvent interactions. This approach separates the problem of determining intermolecular forces from the problem of adding the forces.

For our measurements to be meaningful, the pressure drop across the column must be small. In our experiments, we used capillary columns with estimated pressure drops ranging from 0.2 bars, at a column pressure of 90 bars, to 0.9 bars when operating at 350 bars. In 
addition to small pressure drops, capillary columns have fewer active sites than packed columns.A schematic of the supercritical fluid chromatograph used is shown in Figure 6. We first carried out experiments to check the validity of this technique which involved comparing our results with cosolvent effects reported in the literature. Figure 7 shows the cosolvent effect for anthracene at $35^{\circ} \mathrm{C}$ in $\mathrm{CO}_{2}$ with $3.5 \mathrm{~mol} \%$ methanol added. Our results agree well with those of Dobbs and Johnston (1987). As expected, because there are no specific interactions, the cosolvent effect for nonpolar anthracene is modest. Note that in addition to changing the polarity/polarizability characteristics of the fluid, the addition of cosolvent at constant temperature and pressure usually increases the density of the fluid. Both of these changes, in the absence of other factors, will generally tend to increase the solubility of large solute molecules.

Figure 8 shows the cosolvent effect for 2-naphthol under the same conditions: again our results are consistent with those of Dobbs et al. (1987). In this case, where 2-naphthol and methanol are capable of hydrogen bonding with each other, the cosolvent effect is much larger. We were not able to measure the cosolvent effect at the higher pressures because the fluid mixture became such a good solvent for 2-naphthol that it was essentially unretained in the column.

A variation of looking at cosolvent effects is measuring the ratio of solubilities of a solute in two different fluids; that is, a solvent effect. In Figure 9, the ratio of 2-naphthol's solubility in ethane to its solubility in $\mathrm{CO}_{2}$ is plotted versus pressure at $50^{\circ} \mathrm{C}$. Our results agree well with the literature (estimated from Schmitt and Reid, 1986) despite nearly an order of magnitude change in the ratio. Note that the solvent effect goes from greater than one to less as pressure is increased; this indicates a crossover in the solubility isotherms.

\section{INTERMOLECULAR INTERACTIONS}

The inadequacy of standard equation of state models for SCF solutions may result from the dependence of much of the macroscopic behavior in the highly asymmetric SCF solutions on strong or specific interactions. In this situation, clear understanding of the molecular attractions and repulsions is especially important. Spectroscopic investigations yield insight into the strength of these interactions, and are therefore of fundamental importance when attempting to develop an equation of state that represents SCF solutions with meaningful parameters.

In addition, experimental, theoretical, and computational investigations in SCF solutions suggest that molecular interactions in SCF solutions have led to a growing body of evidence suggesting that in highly asymmetric supercritical mixtures the local environment surrounding solute molecules can differ considerably different from the bulk (Kim and Johnston, $1987 \mathrm{a,b}$; Johnston et al., 1989b; Brennecke and Eckert, 1989 a,b; Brennecke et al., 1996 a,b; Yonker and Smith, 1988, Debenedetti, 1987, Petsche and Debenedetti, 1989; Cochran and Lee, 1989, Wu et al., 1990). These highly asymmetric systems, typical of systems found in SC. applications to coal processing, are characterized by large solute molecules having a relatively large characteristic interaction energy, dissolved in a smaller, more weakly interacting solvent. Much of the evidence suggesting that the local and bulk environment differ significantly in 
SCF solutions has been collected spectroscopically.

Fluorescence spectroscopy was our analytical method of choice for two reasons. First, one can detect the dilute solutions typical of SCFE of heavy organic hydrocarbons, which is often a limitation in absorption. Second, fluorescence spectra are very sensitive to the local solvent environment and this is the region that we were trying to probe (Brennecke and Eckert, 1989b). In fluorescence spectroscopy the dilute sample is irradiated with the appropriate wavelength of ultraviolet light to place some of the molecules in their first excited state. The molecules fluoresce when they emit photons and lose energy back down to the ground state. The fluorescence spectra are relatively insensitive to the exact wavelength of excitation because even if they are promoted into a higher excited vibrational level in the excited electronic state, they quickly lose energy in radiationless processes to the lowest vibrational level before they fluoresce (Lumb, 1978).

\section{SOLUTE/SOLVENT INTERACTIONS}

Solute/solvent interactions were studied in dilute solutions of pyrene in supercritical fluids fluoroform $\left(\mathrm{CF}_{3} \mathrm{H}\right)$, ethylene $\left(\mathrm{C}_{2} \mathrm{H}_{4}\right)$, and carbon dioxide using the fluorescence apparatus shown in Figure 10. Many polycyclic aromatics fluoresce in the ultraviolet-visible range and pyrene in particular has a very high quantum yield. Its spectrum has been welldocumented and it has been used extensively as a probe of solvent environments. The solvents have convenient critical temperatures near ambient: $\mathrm{C}_{2} \mathrm{H}_{4}\left(\mathrm{~T}_{\mathrm{c}}=9.2^{\circ} \mathrm{C}, \mathrm{P}_{\mathrm{c}}=50.4 \mathrm{bar}\right), \mathrm{CF}_{3} \mathrm{H}$ $\left(T_{c}=25.9^{\circ} \mathrm{C}, P_{c}=48.3 \mathrm{bar}\right)$, and $\mathrm{CO}_{2}\left(\mathrm{~T}_{\mathrm{c}}=31.1^{\circ} \mathrm{C}, \mathrm{P}_{\mathrm{c}}=73.8 \mathrm{bar}\right)$. We explored the slightly supercritical region from about $T_{R}=1.0 n 5-1.10$ and $P_{R}=1.01-2.00$. This includes the highly compressible region where the partial molar volumes are extremely large and negative. Solute concentrations were relatively dilute, ranging from $3 \times 10^{-8}$ to $1 \times 10^{-4}$ mole fraction.

The characteristic of the fluorescence spectra that we shall use is unique to some nonfunctional polycyclic aromatics in which the intensity of a forbidden transition is used as a measure of the strength of solute/solvent interactions. The intensity of the forbidden transition can be an exceedingly sensitive measure of the solute/solvent interactions. It is particularly well-documented for pyrene in a wide range of liquid organic solvents. The enhanced band in pyrene is the transition from the lowest vibrational state of the excited electronic state to the lowest vibrational state of the ground electronic state (the 0-0 transition). Nakajima (1971) studied the fluorescence spectra of pyrene in various solvents and attributed the enhancement in the 0-0 transition to the reduction of molecular symmetry in the field of surrounding solvent molecules or to the distortion of the $\pi$-electron cloud by the environmental perturbation. The $0-0$ band (designated $I_{1}$ ) is symmetry forbidden. In contrast, the third peak $\left(\mathrm{I}_{3}\right.$ ) for fluorescence is strong and allowed and relatively solvent-insensitive (Kalyanasundaram and Thomas, 1977). The ratio $I_{1} I_{3}$ is a measure of the local solvent environment because the mechanisms for the disruption of the symmetry mentioned above are nearest-neighbor or firstshell interaction and has been well-documented in various organic liquid solvents (Dong and Winnik, 1982; 1984).

Not only does the nature of the solvent (dipolarity, polarizability, acidity, basicity) affect $I_{1} / I_{3}$, as shown by Kalyanasundaram and Thomas (1977) and Dong and Winnik (1982; 1984), 
but the solvent density changes $I_{1} I_{3}$ (lower densities give lower values of $I_{1} / I_{3}$ ). Clearly, at a reduced temperature of 1.01 , one would have to take into account the strong possibility of local densities that are different than the bulk density in the highly compressible region where the partial molar volumes are very large and negative. However, at temperatures further removed from the critical point, where the compressibility is relatively low and free of anonalies, a plot of $I_{1} / I_{3}$ versus bulk density for a particular solvent might be a good measure of the effect of density on the ratio. We shall use this concept later to determine local densities.

$\mathrm{I}_{1} \mathrm{I}_{3}$ ratios are shown in Figures 11 and 12 for pyrene in $\mathrm{C}_{2} \mathrm{H}_{4}$ and $\mathrm{CF}_{3} \mathrm{H}$. In all cases, $I_{1} / I_{3}$ is higher nearer the critical point when two temperatures at constant density are compared. This indicates much stronger solute/solvent interactions closer to the critical point. For instance, in Figure 12, the ratio $I_{1} I_{3}$ is plotted versus bulk solution density for a $3 \times 10^{-7}$ mole fraction solution of pyrene in SCF fluoroform at $30^{\circ} \mathrm{C}$ and $50^{\circ} \mathrm{C}$. The critical temperature of $\mathrm{CF}_{3} \mathrm{H}$ is $26^{\circ} \mathrm{C}$ so the upper curve is closer to the critical point. The two curves seem to merge at higher pressure, where the compressibility is low and the solvent is very dense. This is what one might anticipate, because at those pressures the solution is at liquid-like densities and the solution is out of the region of large negative partial molar volumes where clustering is most likely to occur.

As mentioned previously, $I_{1} I_{3}$ is a strong function of solveat density. It could be considered measures of local density about the solute, based on the discussion of the mechanisms of forbidden band enhancement. Some clustering may occur even at temperatures far removed from the critical point; in fact, even in attractive liquid mixtures the first shell solvent density is somewhat greater than the bulk density. However, the data at a temperature $20-30^{\circ} \mathrm{C}$ from the critical temperature are the best measures available of the effect of solvent density on $\mathrm{I}_{1} / \mathrm{I}_{3}$. Such is the case for the higher temperature curves in Figures 11 and 12 . The relationship appears to be somewhat linear; however, we know of no theoretical justification for such a fit. In Figure 12, for instance, the lower solid line is a linear least squares fit of the low compressibility points of the $\mathrm{I}_{1} / \mathrm{I}_{3}$ ratio of pyrene in $\mathrm{SCF} \mathrm{CF}_{3} \mathrm{H}$; i.e., the data at $50^{\circ} \mathrm{C}$. It follows that a measure of the density enrichment of the $30^{\circ} \mathrm{C}$ points that are above the line can be obtained by moving horizontally to the line and reading the density. For instance, the local density at point A (bulk density $0.008 \mathrm{gmol} / \mathrm{cc}$ ) may actually be like the local density at the bulk density at point $\mathrm{B}, 0.013 \mathrm{gmol} / \mathrm{cc}$. Using this method, we determined a measure of solvent density enrichment or augmentation of pyrene in $\mathrm{C}_{2} \mathrm{H}_{4}$, and $\mathrm{CF}_{3} \mathrm{H}$. The augmented density is the local density increase over that which would exist without clustering in an equivalent normal liquid of that bulk density. In these experiments the local density is as much as $21 / 2$ times the bulk density. At these conditions the local density is less than at very high pressures (i.e., 1000 psi or more above the critical point) but still very liquid-like in nature.

Even more dramatically, the lower temperature isotherms (nearer the critical temperature) return to the higher temperature lines at very low densities as seen in Figures 11 and 12. These low density data are at conditions where the compressibility is smaller than at slightly higher pressures. The compressibility correlates very well with the infinite dilution partial molar volume so these points are in the same region where $\bar{v}_{2}{ }^{\infty}$ is beginning to sharply decreasing and, subsequently, at a state where one would anticipate less clustering. We 
demonstrate that the clustering does indeed diminish at lower compressibilities in Figures 13 and 14, where the augmented density (local density increase over that in a normal liquid of equivalent bulk density) is shown with the isothermal compressibility for pyrene in supercritical $\mathrm{C}_{2} \mathrm{H}_{4}$ and $\mathrm{CF}_{3} \mathrm{H}$. These values are the difference between the low temperature data points and a linear fit of the higher temperature points. It appears that the highest augmented densities correspond reasonably well with the highest isothermal compressibility fer fluoroform. For ethylene, the compressibility maximum is at a somewhat higher density than the experimental augmented density maximum. The ethylene data are taken at a temperature closer to the critical temperature than $\mathrm{CF}_{3} \mathrm{H}$ so there is more experimental uncertainty in those data, as shown by the considerable scatter in the points in Figure 14. As a result, we believe that further investigations are needed to make conclusive statements about the apparent link between the augmented density and the solvent isothermal compressibility. Invariably, any relationship between augmented density and isothermal compressibility will break down arbitrarily close to the critical point because the solvent compressibility diverges while the augmented density, by its nature, is a finite quantity. Nonetheless, these data indicate that clustering decreases at lower densities where the compressibility decreases, confirming that clustering is most important in the region of high solvent compressibility.

Recent results of molecular dynamics simulations also indicate significant local density enhancement of solvent molecules around solute molecules in SCF solutions. Direct comparison of molecular dynamics results and fluorescence spectroscopy measurements provides the unique opportunity to improve both the interpretation of spectroscopic data and the modelling of solute/solvent interactions in SCF solutions. A direct comparison of local density enhancement in SCF solutions as measured by fluorescence spectroscopy and molecular dynamics simulation yields qualitatively comparable results.

The system studied for this comparison was pyrene in supercritical $\mathrm{CO}_{2}$, at reduced temperatures of 1.02 and 1.14. As previously discussed, the effects of local solvent environment can be quantified using a ratio of pyrene's fluorescence peaks, $I_{1} / I_{3}$. The choice of experimental systems is also limited by constraints on the complexity of the intermolecular potential used in the molecular dynamics simulation. Pyrene, a relatively uncomplicated probe of solute/solvent interactions, satisfies the criteria of both experiments. The system temperatures were chosen based on the desire to study intermolecular interactions near the critical point $\left(T_{R}=1.02\right)$ and the need for a high temperature reference state $\left(T_{R}=1.14\right)$ in calculating local density from pyrene fluorescence. The upper temperature, $T_{R}=1.14$, is the highest temperature for which densities comparable to supercritical fluid densities can be obtained in the fluorescence experiment within the range of allowable pressures.

As previously discussed, the same value $I_{1} / I_{3}$ corresponds to the same local density around the solute molecule, assuming that $\mathrm{I}_{1} / \mathrm{I}_{3}$ is a function of density. Taking the higher temperature as a reference state, the local density enhancement is the difference between the apparent density at $T_{R}=1.02$ and the bulk density. This is determined graphically as the horizontal distance from the lower temperature data to the linear fit of the high temperature data. The density enhancement at $T_{R}=1.02$ determined by this method is shown as the local density/bulk density versus the bulk density in Figure 15.

The molecular dynamics simulations, which are computer-based experiments, are the 
work of Pablo Debenedetti and Irena Petsche at Princeton University. The pyrene/ $/ \mathrm{CO}_{2}$ solution was modelled as a Lennard-Jones fluid using standard mixing rules. The average of the correlation function was determined directly from the simulation for one pyrene molecule in about $1000 \mathrm{CO}_{2}$ molecules. The number of solvent molecules around a solute molecule is calculated by iniegrating the average cor elation function over an arbitrary volume around the solute molecule. For the purposes of this study, the integration volume is the dianeter of one $\mathrm{CO}_{2}$ molecule away from the outer edge of the pyrene molecule, as given by the LennardJones molecular distance parameters. The choice of integration volume affects the absolute value of the calculated density enhancement, but does not affect the qualitative results. The local density enhancement, given as the local/bulk density versus bulk density, is significant at $T_{R}=1.02$, as shown in Figure 16. Local density enhancement is also observed at $T_{R}=1.14$, suggesting that the effect of solute/solvent "charisma" measured from fluorescence spectroscopy is actually greater than observed due to our constrained choice of a high temperature reference.

The local density enhancement around a pyrene molecule in supercritical $\mathrm{CO}_{2}$ as measured by fluorescence spectroscopy and molecular dynamics simulations are very comparable, as shown in Figure 17. The highly satisfactory agreement between these methods, subject to qualitative interpretation, shows great promise for an improved understanding at a molecular level of SCF solutions. Through the comparison of solute/solvent interactions in SCF solutions as measured by significantly different techniques, opportunities exist to improve the interpretation of both the fluciescence spectroscopy data and the molecular dynamics simulation. Better data lead to improved theory. In this way, these results are significant to our modelling work.

\section{SOLUTE/SOLUTE INTERACTIONS}

Solute/solute interactions were investigated through the spectra of excimers, or excited state dimers, in pyrene/SCF systems. Even in liquid solutions, as the concentration of pyrene is increased, the normal fluorescence quantum efficiency decreases, with the corresponding formation of a broad structureless band at longer wavelengths. Extensive studies revealed this to be an excited 1:1 dimer of pyrene in a planar srangement. Although other polycyclic aromatic hydrocarbons form excimers, the particularly high excimer quantum efficiency of pyrene makes it an ideal probe of solute/solute interactions in SCF's. While excimers are not dimers in the sense of a ground state complex, their existence does indicate that there is sufficient interaction in the approximately $10^{-6}$ second lifetime of the excited state to form the excited state complex.

The purpose of this study is to show the importance of solute/solute interactions in SCF mixtures. Indeed, we observe the formation of pyrene excimers even at extremely low concentrations in SCF $\mathrm{CO}_{2}, \mathrm{C}_{2} \mathrm{H}_{4}$, and $\mathrm{CF}_{3} \mathrm{H}$; Figure 18 shows the spectra of pyrene in SCF ethylene at two concentrations. At a mole fraction of just $5.5 \times 10^{-6}$ significant excimer formation takes place, even at high pressures away from the critical point. This mole fraction corresponds to approximately a $5 \times 10^{-5}$ Molar solution. To obtain a similar level of excimer formation in liquid cyclohexane requires a $2 \times 10^{-3}$ Molar solution. One would expect the 
excimer formation to be controlled by diffusion. According to the Einstein-Smoluchowski diffusion theory, the rate should be proportional to the diffusion coefficient, or inversely proportional to the solvent viscosities. This has been confirmed for pyrene in liquids at high pressures. However, the viscosity of cyclohexane at room temperature is $0.88 \mathrm{cp}$ and the viscosity of $\mathrm{SCF} \mathrm{CO}_{2}$ at 150 bar and $35^{\circ} \mathrm{C}$ is $0.074 \mathrm{cp}$. Thus, this could account for a twelve fold increase in excimer formation, but not the forty fold increase observed. It would appear that solute/solute interactions are even more pronounced in asymmetric SCF solutions than in normal liquids.

The solute/solute interactions are important because a large fraction of the solute molecules fluoresce as excimers. In fact, assuming equivalent quantum efficiencies of the normal and excimer fluorescence leads to an estimate of about $50 \%$ of the solute molecules fluorescing as excimers in a solution at a concentration of just $8 \times 10^{-6}$, as shown in Figure 19. Note the increase in the excimer formation at the low bulk densities, which are nearer the critical point. This is most likely due to the dramatically increased diffusivity in the immediate vicinity of the critical point and not to any additional increase in solute/solute interactions. However, the large fraction of molecules fluorescing as excimers strongly suggests that the common practice of neglecting solute/solute interactions in models of SCF phase equilibria when the solute concentration is below 1-2 mole $\%$ is unsubstantiated, and that our concept of infinite dilution in SCF's may need to be reevaluated.

\section{INTERMOLECULAR INTERACTIONS IN COSOLVENT SOLUTIONS}

In addition to soluteisolvent "charisma" we have previously studied, understanding solute/solute and solute/entrainer interactions is very important in the development of a model for all types of practical SCF systems. We have extended our fluorescence spectroscopy technique to the study the entrainer systems naphthalene/triethylamine/ $\mathrm{CO}_{2}$ and 2-naphthol derivatives/alcohol or water cosolvents $/ \mathrm{CO}_{2}$.

Naphthalene/TEA/CO $\mathrm{C}_{2}$. Amines can quench the normal fluorescence of aromatic hydrocarbons by forming fluorescent exciplexes, which result in the appearance of a red-shifted broad, structureless band. In particular, triethylamine effectively quenches the fluorescence of naphthalene solutions in liquid cyclohexane. In terms of SCF solutions, the naphthalene and triethylamine represent the "solute" and "entrainer" respectively, and the exciplex formation can be a measure of the interaction between the solute and entrainer in the highly compressible SCF region.

The normal fluorescence and exciplex formation were recorded for $1 \times 10^{-5}$ mole fraction naphthalene and three concentrations of triethylamine $(0.06$ mole $\%, 0.14$ mole $\%$, and 0.27 mole \%, corresponding to approximately 0.005 to 0.05 Molar) in supercritical $\mathrm{CO}_{2}$ at $35^{\circ} \mathrm{C}$ and $50^{\circ} \mathrm{C}$ and pressures from 74 to 140 bar. All experiments were sufficiently removed from the scaling region, yet some were within the area believed to exhibit the dramatically increased local densities or charisma. Care was taken to remain within the one phase region.

As in previous experiments with pyrene, the results are interpreted in terms of intensity ratios. For naphthalenc, the strength of the solvent interaction is given by the ratio of the first peak to the more stable fourth peak. The plots of $I_{1} I_{4}$ as a function of bulk density for four 
triethylamine concentrations $\left(0.0\right.$ to 0.27 mole \%) are shown in Figures 20 and 21 for $35^{\circ} \mathrm{C}$ and $50^{\circ} \mathrm{C}$ respectively.

Both solvent and triethylamine would be expected to affect the $\mathrm{I}_{1} / \mathrm{I}_{4}$ ratio. In fact, the dramatically decreasing bulk solvent density is the likely cause of the lower $I_{1} / I_{4}$ values at lower bulk densities. At $35^{\circ} \mathrm{C}$, the ratio increases with increased triethylamine mole fraction at all bulk densities, even though the bulk concentration (Molarity) of triethylamine goes down with the decreasing bulk density. The net effect is that at lower bulk densities, nearer the critical point, the presence of triethylamine may contribute a greater fraction of the interactions that disrupt the symmetry and allow the transition to take place. Conversely, at $50^{\circ} \mathrm{C}$ the presence of more triethylamine does not seem to have a significant effect on the ratio. These data are entirely consistent with previous suggestions that the local composition is enriched with the entrainer, especially in the region where the compressibility is high, near the critical point.

The steady state fluorescence measurements showing exciplex formation in the system naphthalene/triethylamine(TEA)/ $\mathrm{CO}_{2}$ indicate a substantial pressure effect on the rate constant for exciplex formation in the near critical region. Using a proposed mechanism for the fluorescence of naphthalene in the presence of triethylamine (Van and Hammond, 1078), the ratio of quantum yields in the presence and absence of TEA can be related to the rate constants and concentration of triethylamine. The quantum yield can be estimated by the area under the fluorescence peak and in Figure 22 the ratio of normal fluorescence area of naphthalene without and with trieth"lamine is shown as a function of TEA concentration at $35^{\circ} \mathrm{C}$.

In the thermodynamic framework of transition state theory the rate of a bimolecular reaction will be a function of pressure, given by

$$
\frac{\partial \ln k}{\partial P}=-\Delta v^{*} / R T
$$

where $\Delta \mathrm{v}^{\neq}$is the activation volume, given by the difference in partial molar volumes of the transition state and the substrates. In the mechanism, the only bimolecular reaction is the formation of the exciplex from an excited naphthalene molecule and a TEA molecule with rate constant $\mathrm{k}_{3}$ and the difference in partial molar volumes may be significant so we will concentrate our efforts on this reaction. Unfortunately, the data do not give the pressure dependence of $k_{3}$ but rather that of a complicated ratio of rate constants. However, with the assumption that only $k_{3}$ depends on pressure, the natural logarithm of the slopes in Figure 22 is just the logarithm of $k_{3}$ plus a constant and therefore $\partial(\ln ($ slopes from Figure 1))/วP should

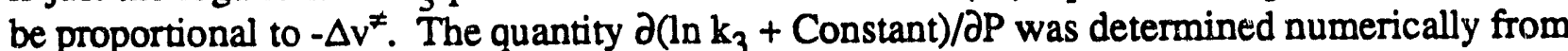
the values in Figure 22 and is plotted as a function of pressure in Figure 23 for $35^{\circ} \mathrm{C}$. Notice the sharp peak which is in the highly compressible region nearer the critical point.

The activation volume can be estimated with an equation of state by calculating the partial molar volumes of substrates and complex. We used the Peng-Robinson equation to estimate the equation of state contribution to the partial molar volumes and also included a term due to electrostriction of the solvent about the substrates and complex. Calculated activation volumes are shown in Figure 24 for $35^{\circ} \mathrm{C}$ and $50^{\circ} \mathrm{C}$. The contribution of the 
electrostriction term is overwhelming and causes the activation volumes to be large and negative, especially in the compressible region at $35^{\circ} \mathrm{C}$. Since $\partial \ln \mathrm{k}_{3} / \partial \mathrm{P}$ is proportional to the negative of the activation volume, the calculations predict a large positive peak in $\partial \ln k_{3} / \partial P$ in the region of highest compressibility which is in remarkable agreement with the experimental data. This analysis suggests that taking the pressure effect on the reaction rate is not only appropriate but also necessary when looking at reactions in highly compressible SCF's.

2-Naphthol Derivatives/Cosolvent SCF Systems. Another probe of local compositious around a solute, proton transfer from 2-naphthol was chosen since it has been shown to be sensitive to solvent structure in liquids. Naphthols are useful probe molecules due to the large change in acidity of the proton upon excitation (Ireland and Wyatt, 1976). The $\mathrm{pK}_{\mathrm{a}}$ of 2naphthol is approximately 9.5 in the ground state and 2.7 in the excited state making it a fairly strong photo-acid (Tsutsumi and Shizuka, 1980). The solvent effect on proton transfer is shown schematically in Figure 25, where the solvated neutral naphthol is the most stable species in the ground state. However, with the correct solvent structure in the excited state, the proton may be solvated and the naphtholate anion may become more stable. This stabilization of the anion species in solution is sensitive to the orientation of solvent around the solute as determined from studies in liquid solvents (Lee, et al., 1985). The dissociation of 2-naphthol is specific for water and is apparently independent of the dielectric constant of the solvent (Huppert, et al., 1982). Finally, a random walk solvation model which incorporates the probability of forming water clusters of specific sizes around the naphthol implies that a minimum cluster size of four water molecules is required to facilitate proton transfer (Lee, et al., 1986).

In supercritical fluids, the possibility of local composition enhancements of cosolvent about a solute suggests that we should see enhancement of anion fluorescence if the water cosolvent clusters effectively about the 2-naphthol solute. Although in liquids the water concentration must be $>30 \%$ to see anion emission, the higher diffusivity and density fluctuations in SCF's could allow stabilization of the anion at much lower water concentrations provided that the water molecules provide sufficient structure. Therefore the purpose of these experiments was to investigate 2-naphthol fluorescence in supercritical $\mathrm{CO}_{2}$ with water cosolvent in the highly compressible region of the mixture to probe the local environment about the solute.

Experiments were carried out in supercritical $\mathrm{CO}_{2}$ with 0.003 mole fraction water at 35 and 50.C over a range of pressures which corresponded to specifically chosen density values. Our objective was to sample the regions above and below the critical density through the region of high compressibility since this is the region where maximum cosolvent composition enhancement would be expected.

The effect of water cosolvent on the emission of 2-naphthol is shown in Figure 26. There is some loss of fine structure but no detectable peak in the $400-500 \mathrm{~nm}$ range indicative of the anion species. This lack of anion emission is likely due to the low concentration of water in the solution, and since the concentration is limited by the water $/ \mathrm{CO}_{2}$ phase equilibria there is a need for a more sensitive probe.

Cyano-naphthol is a more sensitive probe to water due to its increased excited state 
acidity. An additional benefit of cyano-naphthol as a proton transfer probe is that the anion species is present in pure alcohols; not only will the probe be more sensitive to water but we may also observe proton transfer for $\mathrm{CO}_{2}$ doped with methanol, which has a much higher solubility than water.

The effect of water ( 0.003 mole fraction) and methanol ( 0.02 mole fraction) cosolvents on the emission of $5 \mathrm{CN} 2 \mathrm{~N}$ is shown in Figure 27. For this probe there is complete loss of fine structure, a significant red shift in the neutral emission with methanol cosolvent, but again, no detectable anion emission.

The red shift of the $5 \mathrm{CN} 2 \mathrm{~N}$ emission due to methanol was determined relative to the second peak in the pure $\mathrm{CO}_{2}$. The shift is shown in Figure 28 for two temperatures over the range of density studied. The reported mixture densities were found by multiplying the pure $\mathrm{CO}_{2}$ density (interpolated from IUPAC data) by a calculated ratio of mixture to pure density. The ratio was calculated from the Soave-Redlich-Kwong equation of state using a binary interaction parameter $\left(\mathrm{k}_{12}\right)$ which had been fit to $\mathrm{CO}_{2}$ - methanol VLE data at the temperature of interest. The parameters were 0.12 at $35 \mathrm{C}$ and 0.14 at $50 \mathrm{C}$. Although the scatter in the data is slightly larger than the estimated uncertainty, there appears to be no clear dependence on either temperature or density which indicates that the thermodynamic properties of the solvent have little effect on the cosolvent/solute interaction in this case.

We suggest the following possible reasons for the absence of anion emission in these experiments: First, it is possible for water and methanol to form adducts with $\mathrm{CO}_{2}$ which might inhibit their ability to solvate the proton. This possibility was checked using water as a cosolvent in supercritical ethylene, which will not interact with water, data do not indicate formation of an anion therefore solvent/cosolvent adducts are not a plausible explanation. Second, perhaps the anion is being formed but is quenched very efficiently. The anion species would be more susceptible to quenching than the neutral molecule. This possibility could be investigated in principle with fluorescence lifetime studies, but this has not been done. It is entirely possible that the anion emission is so weak as to be hidden underneath the tail of the neutral emission.

Finally, coupled with the phase equilibrium problem of concentrating the cosolvent, perhaps the composition of cosolvent around the probe is not sufficient to facilitate proton transfer. This implies that if the cosolvent is indeed "clustering" around the solute, there is insufficient structural integrity to solvate the proton.

Computer simulation visualizations (Petsche and Debenedetti, 1989) of local density augmentation show that the "clustering" is a dynamic process, with solvent molecules interchanging fairly rapidly in close proximity of the solute. Calculated viscosities are slightly higher in the region around the solute, and the diffusivity in SCF's is approximately two orders of magnitude greater than in liquids. Also, even with local density augmentation, the local density at the surface of the solute molecule is enough less than that in a liquid to create a very substantial increase in free volume, with a concomitant decrease in structure. If this is the case, a more sensitive probe molecule is needed to observe local composition enhancement.

There are a number of experiments using the proton transfer mechanism which may yet yield information regarding local compositions. A more polar solvent such as fluoroform may be able to support the proton in solution more easily than the nonpolar solvents. Conversely, 
since information about the solute environment is desired, the real challenge is identifying probe molecules that are both sensitive to the small amounts of cosolvent and follow relatively simple photophysical kinetics.

\section{Chemical-Physical Modelling of Molecular Charisma and Cosolvent Effects}

Developing an equation of state (EOS) that models PVT and solubility behavior accurately in supercritical fluids (SCF's) is challenging; most models give quantitative results only when several adjustable parameters are introduced. Nevertheless, accurate models are vital to the design and evaluation of supercritical fluid processes. There is a pressing need for a better understanding of the molecular level interactions in supercritical fluid solutions.

In the presence of cosolvents, solubilities of solutes may increase an order of magnitude or more relative to the pure SCF. In many cases, this increase cannot be explained solely by dispersion forces; there must be specific interactions between the solute and cosolvent, such as hydrogen bonding or charge transfer coinplex formation. Specific interactions are usually strong and saturable, and result in the formation of compounds. The commonly used cubic EOS's are based on mean field attractive interactions that are modest and nonsaturable; thus, they cannot account for specific interactions.

Chemical models, which date from the early work of Dolezalek (1908), invoke the law of mass action but have generally found less favor than physical models. They are more specific and some knowledge of the chemistry of the solution is necessary to apply them. Furthermore, they are substantially more complex mathematically, especially as solutions go from binary to multicomponent, and the parameters are more difficult to determine. Pure chemical models do not address the physical interactions between molecules, which are often equally as important as chemical interactions.

In this work, we couple a simple cubic EOS with a chemical term that can describe specific interactions. Depending on the nature of the chemical term, it can introduce several new adjustable parameters. Here, we reduce the number of modeling parameters by using a group contribution technique to linearize the Gibbs energy of formation of compounds in solution. Further, we discuss the possibility of predicting the model parameters by using spectroscopic measurements or properties of the interacting molecules. Next, we apply the model to supercritical fluid solutions. Not only can an association model be applied to cosolvent solution, but it may be used to describe clustering of the solvent molecules around the solute in the critical region.

A chemical term was developed for solvation of species in solution. However, first we shall discuss briefly the components of an EOS. A typical EOS is

$$
\frac{P V}{n R T}=1+Z_{\text {rep }}+Z_{a n}
$$

where $P$ is the pressure, $V$ is the volume, $n$ is the number of moles, $R$ is the gas constant, $T$ is the temperature, and $Z_{\text {rep }}$ and $Z_{\text {att }}$ are the contributions of repulsive and attractive intermolecular forces, respectively, to the compressibility factor. Usually, the EOS contains 
at least two parameters, an energy parameter, $a$, and a size parameter, $b$. In a mixture, the mixture parameters are related to the pure component parameters by mixing rules, commonly

$$
\begin{gathered}
a_{m d x}=\sum_{i} \sum_{j} y_{i} y_{j}\left(a_{i} a_{j}\right)^{1 / 2}\left(1-k_{i j}\right) \\
b_{m b x}=\sum_{i} y_{i} b_{i}
\end{gathered}
$$

where $y_{i}$ is the mole fraction of component $i$ and $k_{i j}$ is an adjustable binary parameter usually necessary to fit experimental data quantitatively. When $i=j$, $k_{i j}=0$.

In general, in a solution consisting of components $A$ and $B$ with apparent composition $y_{A}$ and $y_{B}$, a solvated species has the formula $A_{i} B_{j}$ where $i$ and $j$ are integers from one to infinity. In the presence of compound formation, the true mole fractions are different than the apparent mole fractions; these are denoted by $z_{A}$ and $z_{B}$ for the monomeric species $A$ and $B$, and $z_{i j}$ for the molecular complex $A_{i} B_{j}$. The apparent number of moles in solution is $n_{0}$, and the true number of moles is $n_{T}$, the ratio $n_{T} / n_{0}$ is indicative of the degree of solvation. When $n_{T} / n_{0}=1$, there is no solvation; as solvation increases, $n_{T} / n_{0}$ decreases. If only $1: 1$ complexes exist in solution, $n_{T} / n_{0}=0.5$ with complete solvation; however, if complexes with more than two molecules form, $n_{T} / n_{0}$ can be even smaller, approaching zero as an extreme case.

One of the decisions that must be made when developing a chemical model is which compounds exist in solution. Determining the existence of compounds can be fairly straightforward in some cases such as where intermetallic compounds are known in some liquid metal solutions (Hultgren et al., 1973); however, to date in supercritical fluid solutions, there are no data showing the existence of any particular compound. This necessitates choosing the compounds in solution either arbitrarily or from estimates based on knowledge of solution chemistry. Here, two models are developed: infinite solvation and 1:1 complex formation.

In the infinite solvation model, every species $A_{i} B_{j}$ is assumed to exist and is at equilibrium with the monomers

$$
i A+j B \sim A_{t} B_{j}
$$

The equilibrium constant for this reaction, $K_{i j}$, is defined as

$$
K_{l j}=\frac{z_{l j}}{z_{A}^{\prime} z_{B}^{j}} \frac{\phi_{l j}}{P^{i+j+1} \phi_{A}^{\prime} \phi_{B}^{\prime}}
$$

where $\phi$ represents fugacity coefficients. When defined this way, $K_{i j}$ is dependent on temperature only. Because there are an infinite number of equilibrium reactions occurring, this introduces an infinite number of adjustable parameters to the model; thus, it is necessary to use some means to reduce the number of parameters. Ideal chemical theory assumes that the fugacity coefficients are unity, however this is not realistic in a supercritical fluid solution. Determination of the fugacity coefficients requires a physical model, such as a cubic EOS which in turn requires size and energy parameters for all of the species in solution. In 
addition, since the fugacity coefficients are a function of the true mole fractions, the problem is highly coupled mathematically. These concerns are addressed below.

Here, the number of chemical parameters is reduced by adopting a group contribution technique first employed by Howell et al. (1988). They observed that the Gibbs energy of formation for liquid metallic compounds in a particular binary system is proportional to the number of atoms comprising the compounds

$$
g_{X}^{f}=\left(n_{X}-1\right) G
$$

where $g_{X} f$ is the Gibbs energy of formation for compound $\mathrm{X}$, and $n_{X}$ is the number of atoms in $X . G$ is then a single, adjustable parameter that can be used to determine all of the equilibrium constants in a particular binary solution. We extend this treatment to a supercritical fluid solution by assuming that the Gibbs energy of formation of a complex of molecules, $A_{i} B_{j}$ is proportional to the number of molecules in the complex. With this assumption, the equilibrium constants are

$$
K_{u}=\exp \left[\frac{G(i+j-1)}{R T}\right]
$$

thus reducing an infinite number of adjustable parameters to one.

Ikonomou and Donohue (1988) developed an infinite equilibria model for their chemical term, but stated that they assumed the equilibrium constants, $K_{i j}$, were all equal. However, because of an error in the mathematics, it turns out that they inadvertently linearized the Gibbs energy of formation of the chemical complexes so that the $K_{i j}$ 's were not equal.

Next, to reduce the mathematical complexity it is necessary to choose carefully the equation of state parameters for the complexes and the mixing rules (Heidemann and Prausnitz, 1976; Hu et al., 1984). A reasonable assumption for the EOS size parameter for $A_{i} B_{j}, b_{i j}$, is that it is the sum of the size parameters of the monomers in the complex; that is,

$$
b_{i j}=i b_{A}+j b_{B}
$$

where $b_{A}$ and $b_{B}$ are the size parameters for the monomeric species. When this is combined with the usual mixing rule for size parameters, one obtains

$$
b_{m i x}=\left(\frac{n_{0}}{n_{T}}\right)\left(y_{A} b_{A}+y_{B} b_{B}\right)
$$

that is, the size parameter for the solvated mixture is merely the product of the apparent size parameter for the mixture and the ratio $n_{0} / n_{T}$.

The situation for the EOS energy parameter is somewhat more complicated. Heidemann and Prausnitz (1976), when looking at mixtures with one self-associating species, proposed that the energy parameter for the $j$-mer be equal to $f^{a}$ where $a$ is the energy parameter of the monomer. Hu et al. (1984) found that this overestimates the effect of $j$ on the energy parameter of the complex; however, the alternative is to add another adjustable parameter. With this assumption, the mixing rule for the energy parameter, equation (12), shows that the 
mixture energy parameter is the product of the square of $n_{T} / n_{0}$ and the apparent mixture energy parameter. For our treatment of solvated solution, we adopt a parallel approach. The energy parameter for species $\mathrm{A}_{i} \mathrm{~B}_{j}, a_{i j}$, is

$$
a_{i j}=i j\left(a_{A} a_{B}\right)^{1 / 2}
$$

where $a_{A}$ and $a_{B}$ are the energy parameters for the monomers. Unfortunately, when this expression is combined with the normal mixing rule for energy parameters, a very complicated expression is the result. Instead, we shall use the same expression obtained by Heidemann and Prausnitz

$$
a_{\operatorname{mix}}=\left(\frac{n_{0}}{n_{T}}\right)^{2}\left[y_{A}^{2} a_{A}+2 y_{A} y_{B}\left(a_{A} a_{B}\right)^{1 / 2}\left(1-k_{A B}\right)+y_{B}^{2} a_{B}\right]
$$

where $k_{A B}$ is the ubiquitous binary interaction parameter. Both of the mixing expressions involve the apparent mixture parameters, which are easily caiculated, and the ratio $n_{T} / n_{0}$; addition of an inert (non-solvating) component to the mixture does not change this situation.

With these choices for the complex parameters and mixing rules there are two propitious results. First, the ratio of fugacity coefficients in the equilibrium expression does not depend on the true mole fractions, greatly simplifying the mathematics. Second, upon substitution of the new mixing rules into the equation of state it becomes

$$
\frac{P V}{n_{0} R T}=\frac{n_{T}}{n_{0}}+Z_{\text {rep }}+Z_{\text {att }}
$$

with apparent mixture parameters used in the EOS. Incredibly, the only effect of solvation on the equation of state is the change in the ideal gas term.

Here, we use the Soave-Redlich-Kwong equation of state (SRK, Soave, 1972) for the physical portion of the model. In a solvated solution, it is

$$
P=\frac{n_{T}}{n_{0}} \frac{R T}{(v-b)}-\frac{a}{v(v+b)}
$$

where $v$ is the apparent molar volume. With this choice, the ratio of fugacity coeificients in the equilibrium expression (15) can be determined (for details of this, see the Appendix in $\mathrm{Hu}$ et al. (1984)). Upon substituting equation (17) for $K_{i j}$ and solving for $z_{i j}$, one obtains

$$
z_{i j}=z_{A}^{i} z_{B}^{J}\left(\alpha K_{A B}\right)^{(i+j-1)}
$$

where, for simplicity, $\alpha$ and $K_{A B}$ have been defined as

$$
\alpha=\frac{n_{T} R T}{n_{0}(v-b)}
$$

and 


$$
K_{A B}=\exp \left(\frac{G}{R T}\right)
$$

Three material balances can be written; the first is that the sum of the true mole fractions must be one

$$
1=z_{A}+z_{B}+\sum_{i=1}^{\infty} \sum_{j=1}^{\infty} z_{1 j}+\frac{n_{0}}{n_{T}} y_{I}
$$

Included here is the presence of a component (or components) $\mathrm{I}$, present at apparent mole fraction $y_{i}$, which does not participate in the solvation reactions. Next, balances for A and B can be formulated

$$
n_{0} x_{A}=n_{T}\left(z_{A}+\sum_{i=1}^{\infty} \sum_{j=1}^{\infty} i z_{V}\right)
$$

with a similar balance for $B$. Upon using equation (14) for $z_{i j}$ in the three balances, the infinite sums converge and can be put into closed form easily

$$
\begin{gathered}
1=z_{A}+z_{B}+K_{A B} \alpha \frac{z_{A}}{\left(1-K_{A B} z_{A} \alpha\right)} \frac{z_{B}}{\left(1-K_{A B} z_{B} \alpha\right)}+y_{1} \frac{n_{0}}{n_{T}} \\
x_{A} \frac{n_{0}}{n_{T}}=z_{A}+K_{A B} \alpha \frac{z_{A}}{\left(1-K_{A B} \alpha z_{A}\right)^{2}} \frac{z_{B}}{\left(1-K_{A B} \alpha z_{B}\right)} \\
x_{B} \frac{n_{0}}{n_{T}}=z_{B}+K_{A B} \alpha \frac{z_{A}}{\left(1-K_{A B} \alpha z_{A}\right)} \frac{z_{B}}{\left(1-K_{A B} \alpha z_{B}\right)^{2}}
\end{gathered}
$$

There are three equations in three unknowns: the true mole fractions of the monomeric species, $z_{A}$ and $z_{B}$, and the degree ratio $n_{T} / n_{0}$. Note that $\alpha$ contains the ratio $n_{T} / n_{0}$.

In cases such as hydrogen bonding in solution, it is probably more appropriate to assume the formation of $1: 1$ complexes

$$
A+B-A B
$$

with equilibrium constant $K_{A B}$. This model is the same as the infinite equilibria model except for the mass balances; for the binary model, they are

$$
\begin{gathered}
z_{A}+z_{B}+K_{A B} z_{A} z_{B} \alpha+\frac{n_{0}}{n_{T}} y_{I}=1 \\
n_{0} x_{A}=n_{T}\left(z_{A}+K_{A B} z_{A} z_{B} \alpha\right)
\end{gathered}
$$




$$
n_{0} x_{B}=n_{T}\left(z_{B}+K_{A B} z_{A} z_{B} \alpha\right)
$$

The algorithm to solve the equation of state at given $T, P$, and $y$ is to guess $n_{T} / n_{0}$, solve equation (13) for $v$, then solve the mass balances, giving a new guess of $n_{T} / n_{0}$ which can be checked for convergence. Often, one wants to calculate solubilities from an EOS; this involves guessing $y$, solving the EOS as described above, then calculating the fugacity of the solute in the SCF phase and comparing it with the solid phase fugacity of the solute

$$
P_{i}^{\text {vap }} \exp \left(\frac{v_{i}^{\text {mol } P}}{R T}\right)=z_{i} \phi_{i} P
$$

where $P^{\text {vap }}$ is the vapor pressure of the solute at $T, v^{\mathrm{mol}}$ is its molar volume, and $\phi$ is the fugacity coefficient of the monomer determined from the EOS as follows

$$
\begin{aligned}
& \ln \phi_{i}=\ln \left(\frac{v}{\left(v-b_{m \times x}\right)}\right)+\frac{n_{T}}{n_{0}} \frac{b_{1}}{\left(v-b_{m d x}\right)}-\frac{2 \sum_{j} y_{j} a_{i j}}{b_{m x x} R T} \ln \left(\frac{v+b_{m \alpha x}}{v}\right) \\
& +\frac{a_{m a x} b_{1}}{b_{m i x}^{2} R T}\left[\ln \left(\frac{\left(v+b_{m d x}\right)}{v}\right)-\frac{b_{m+x}}{\left(v+b_{m d x}\right)}\right]-\ln \left(\frac{P v}{R T}\right)
\end{aligned}
$$

Iteration is continued until the fugacities are equal yielding the solubility predicted by the EOS.

Now we examine the use of the infinite equilibria model to describe "molecular charisma" in a solid-SCF system and then the binary model to examine cosolvent effects in supercritical fluid solutions.

Solid-SCF system. Through progress made over the past few years, it appears that the molecular-level structure of a supercritical fluid is quite different than that of a liquid or a gas. Experimental studies (Eckert et al., 1986b; Kajimoto et al., 1988; Brennecke et al., 1990a,b), computer simulations (Petsche and Debenedetti, 1989), and direct calculations of pair correlation functions (Cochran and Lee, 1989) indicate that the local density of solvent molecules around a solute molecule can be significantly greater than the bulk density, a phenomenon known as molecular charisma.

Debenedetti (1987) used a fluctuation analysis to derive an expression for the solute infinite dilution partial molar volume in terms of an actual cluster size of solvent molecules around a solute. Cluster sizes determined in this way from experimental partial molar volume data indicate as many as 100 solvent wolecules around a solute in the highly compressible region. Although the environment around the solute is quite dynamic (Tomasko et al., 1991), it may be appropriate to treat the cluster as a sort of chemical complex. Because many solvent molecules and more than one solute molecule may be associated in such a cluster, the infinite equilibria model is most appropriate. Because the solubility of naphthalene in $\mathrm{SCF}_{2}$ is well known, we modelled this system.

The pure component SRK parameters for $\mathrm{CO}_{2}$ were obtained from Morris and Turek 
(1986), who used vapor pressure and volume data to fit the temperature dependence of the parameters. The parameters for naphthalene were determined from liquid vapor pressure data (Fowler et al., 1968) using the normal SRK temperature dependence. The solubility data modeled were those of Tsekhanskaya et al. (1964) which have been found by many subsequent researchers to be accurate. In fitting the solubility data, the objective function that was minimized was

$$
f=\sum\left(\frac{\left|y_{\text {calc }}-y_{\exp }\right|}{y_{\exp }}\right)^{2}
$$

where $y_{\text {calc }}$ is the solubility calculated from the equation of state, and $y_{\exp }$ is the experimental solubility. Usually, the optimized parameters were sensitive to the objective function.

Figure 29 shows the solubility of naphthalene in $\mathrm{CO}_{2}$ at $45^{\circ} \mathrm{C}$ along with the best fit for the SRK EOS and the chemical-physical model. The average error for the SRK was $9.6 \%$ compared with $8.6 \%$ for the chemical-physical model. The majority of the error was at the lower pressures, nearer the critical region. This is not an entirely fair comparison, however, as there was only one adjustable parameter for the SRK $\left(k_{i j}=0.121\right)$ versus two for the chemphys model $\left(k_{i j}=0.139\right.$ and $\left.K_{i j}=2.08 \times 10^{-5} \mathrm{psi}^{-1}\right)$. (Note that $k$ represents a physical interaction and $K$ a chemical one.) The small size of the improvement in the fit is partially explained by the fact that $k_{i j}$ and $K_{i j}$ in some ways represent the same phenomena, as explained below.

For asymmetric systems, such as those generally found in SCF solutions, the geometric mean mixing rule for energy parameters overpredicts the cross energy parameter (Hudson and McCoubrey, 1960). The binary interaction parameter, $k_{i j}$, is the deviation of the cross energy parameter, $a_{i j}$, from the geometric mean of the pure component energy parameters $a_{i}$ and $a_{j}$. The larger that $k_{i j}$ becomes, the less the physical attraction between $i$ and $j$ becomes. On the other hand, an increase in $K_{i j}$, which also indicates the interaction energy between $i$ and $j$, increases the attraction energy. This explains why $k_{i j}$ is larger for the chemical-physical model than the pure SRK; as one "turns on" chemical interactions, the physical interactions must be diminished. Note that for the pure SRK, $k_{i j}$ is positive; this indicates that there is no large specific interaction between $\mathrm{CO}_{2}$ and naphthalene. In any event, both of these parameters adjust the interaction energy between unlike molecules, thus are not independent of each other. If, instead of adding the equilibrium constant as a second adjustable parameter, a parameter adjusting the cross size term is introduced, the errors in predicting the solubility for the $\mathrm{CO}_{2}$ naphthalene system are reduced to $2.9 \%$.

It was also observed that if the equilibrium constant was above a certain level, depending on the highest pressures fit in the solubility isotherm, the chemical-physical model did not converge. This was attributed to a "black-hole" type effect; as the density of the fluid increased above a critical level, solvent molecules collapsed around the solute in tremendous numbers, basically creating a solution with only a few, huge molecular complexes in it. It is not known whether this is a true feature of the model, or if small errors due to truncation in the computer becanie magnified into this effect.

Similar results were obtained for other nonpolar solutes; that is, relatively small 
improvements in fitting solubility data upon addition of a chemical term. It appears that from a computational point of view, it is better to add an adjustable size parameter rather than a chemical equilibrium constant because it gives a better fit and is much simpler mathematically. From a physical point of view, the advantage of a chemical term is that the potential exists for the equilibrium constant to be measured from spectroscopic data as has been done for liquids (Eckert et al., 1986a; Karachewski et al., 1989); in addition, the temperature dependence of an equilibrium constant has a theoretical basis contrary to $k_{i j}$. Nevertheless, it does not appear that a chemical term describes the physics of solvation of a nonpolar solute in a SCF better.

Cosolvent System. As discussed in previous chapters of this thesis, very large solubility enhancements have been observed in SCF's in presence of cosolvents. Clearly, in some cases this is due to specific interactions between the solute and cosolvent. Here we use the simple binary equilibrium model $(A+B+A B)$ coupled with the $S R K$ equation of state to examine cosolvent effects.

As above, the EOS parameters for the SCF's were obtained from Morris and Turek (1986). The cosolvent parameters were optimized to fit liquid vapor pressure data, and the physical interaction parameter, $k_{i j}$, between the SCF and cosolvent was fit to VLE data, if available, or set to 0.10 (the results of the model were not very sensitive to this parameter in the pressure and temperature ranges studied). Because critical properties and liquid vapor pressure data for the solutes were unreliable, the SRK $a$ and $b$ parameters for the solutes were acquired by fitting them to solid-SCF data with $k_{i j}=0.10$. The $k_{i j}$ for the cosolvent-solute interaction was set arbitrarily to 0.10 .

We began by looking at the solutes carbazole and 2-naphthol in ethane with the cosolvent ethanol. Both of these solutes are good hydrogen bond donors and the ethanol is a good acceptor; the reverse interaction is also possible in both cases, but less likely (Ekart et al., 1992). The cosolvent effecis for both of these solutes is large, making probable the possibility of specific interactions. The data fit were at $50^{\circ} \mathrm{C}$ at pressure from 100 to 200 bars, and cosolvent compositions from 0.8 to $9.5 \mathrm{~mol} \%$ ethanol.

The results for carbazole and 2-naphthol are shown in Figures 30 and 31; the model does a fairly good job of fitting the carbazole data. On the other hand, the model significantly overpredicts the cosolvent effect for 2-naphthol at the higher concentrations of ethanol. In both cases, the cosolvent effects for the lower compositions tend to be underpredicted and the higher compositions overpredicted. In other words, the composition dependence of the cosolvent effect predicted by the model is too strong. This may be caused by a flaw in the model, but there are other possibilities that are physically sound.

More than likely, not all of the cosolvent effect is due to the dispersion forces, described by the physical EOS, and hydrogen bonding, described by the chemical term; there are also dipole-dipole and dipole-induced dipole forces not described by the model. In addition, we are not including the self-association of the alcohols. As the alcohol concentration increased, more of it would be involved in hydrogen-bonds with itself, not available for the solutes. Finally, at lower concentrations of alcohol, it is possible that there is enhanced composition of the alcohol in the solute environment relative to the bulk. If so, the equilibrium constant would be overpredicted at the lower compositions during fitting.

The model was also used to correlate data for 2-naphthol and carbazole with other 
cosolvents. For the cosolvents 1,1,1-trichloroethane and 1,2-dibromoethane, the equilibrium constants are very small or negative. (Of course, a negative equilibrium constant has no physical meaning, but it indicates that the physical $k_{i j}$ is not large enough.) This is expected as these cosolvents have no hydrogen bonding ability. A purely physical model, such as the SRK EOS, can fit the data for these cosolvents with reasonable parameters.

Anthracene is a nonpolar molecule, not capable of donating or accepting protons. When the chemical-physical model is applied to cosolvent effects for anthracene, the optimum equilibrium constant is very small or negative in every case. Again, this indicates that in the absence of specific interactions, a typical cuoic EOS can fit cosolvent effects.

\section{SUMMARY}

Supercritical fluids show potential for a variety of coal processing applications. To determine the phase behavior and provide a basis for modelling representative coal compounds in SCF solutions, we have assembled a solubility database for these compounds in SCF's. With the goal of developing an equation of state that models SCF solutions with chemicallymeaningful parameters, we have also investigated intermolecular interactions and cosolvent effects in these systems.

We have extended the solubility database of model coal compounds in SCF solutions to higher temperature, as well developing two novel techniques for measuring solubilities in supercritical fluids. A modified UV-vis spectrophotometer is capable of solubility determinations for UV active solutes over a large range of solubility limits. A rapid SCF chromatographic technique was developed to measure solubilities and cosolvent effects in SCF solutions and was tested with nonpolar and polar solutes. Our results agree well with the literature, suggesting that for these solutes the change in retention due to the presence of cosolvent is attributable to interactions in the fluid phase.

From spectroscopic investigations wo obtained information on molecular interactions in SCF solutions that cannot be obtained from bulk phase properties. Thrcugh fluorescent spectroscopy, we were able to probe sclute/solute, solute/solvent, and solute/cosolvent interactions in SCF systems. The $I_{1} / I_{3}$ ratios in the fluorescence spectra of pyrene in supercritical ethylene, fluoroform, and carbon dioxide indicated much stronger solute/solvent interactions nearer the critical point, suggesting that the local and bulk density around a solute differ appreciably. A direct comparison of augmented densities in SCF carbon dioxide determined by molecular dynamics simulation and fluorescence spectroscopy gave very satisfactory results. The spectroscopic investigation of intermolecular interactions in the naphthalene/triethylamine/ $\mathrm{CO}_{2}$ cosolvent system is also consistent with an enhanced local composition around the solute molecule.

The formation of pyrene excimers in SCF solutions, measured using fluorescence spectroscopy, was used to study solute/solute interactions. From this study, it would appear that solute/solute interactions are even more pronounced in asymmetric SCF solutions than in normal liquids.

We have used a proton transfer known to be sensitive to water as a mechanism to probe 
the cosolvent composition around a solute in supercritical fluids and discern any difference between local and bulk concentrations. No proton transfer was observed from either 2naphthol or 5-cytno-2-naphthol, presumably indicating insufficient structure in the SCF to solvate the proton. Although significant cosolvent effects on the fluorescence emission were observed, these appear to be independent of the thermodynamic variables.

Using our solubility database and knowledge of intermolecular interactions, we have developed a simple chemical-physical model to describe solute-solvent and solute-cosolvent interactions in SCF solutions. For solute-solute interactions, we used a new infinite equilibria model based upon a linearization of the Gibbs energy of formation. It did not perform significantly better than a pure physical model, but if chemical equilibrium constants can be measured independently, there may be no need for adjustable parameters. 


\section{PUBLICATIONS}

Brennecke, J. F.; Eckert, C. A., "Phase Equilibria for Supercritical Fluid Process Design," AIChE J. 1989, 35(9), 1409.

Brennecke, J. F.; Eckert, C. A., "Fluorescence Spectroscopy Studies of Intermolecular Interactions in Supercritical Fluids," ACS Symp. Series 406., K. P. Johnston and J. M. L. Penninger, eds. 1989, 14.

Brennecke, J. F.; Tomasko, D.L.; Eckert, C. A., "Pyrene Excimer and Naphthalene/Triethylamine Exciplex Formation in Supercritical Fluid Solutions," J. Phys. Chem., 1990, $94,7692$.

Brennecke, J.F., Tomasko, D.L., Peshkin, J., and Eckert, C.A., "Fluorescence Spectroscopy Studies of Dilute Supercritical Solutions," I\&EC Res., 1990, 29, 1682.

Eckert, C.A.; Ekart, M.P.; Brennecke, J.F., "Molecular Analysis of Phase Equilibria in Supercritical Fluid Solutions," pp. 165-192, CRC Press, J. Ely and T. Bruno, Eds, 1991.

Ekart, M.P.; Bennett, K. L.; Eckert, C.A., "A Chromatographic Technique to Measure the Effects of Specific Interactions in Supercritical Fluid Solutions," ACS Symposium Series, submitted, 1991.

Ekarh, M.P., K.L. Bennett, S.M. Ekart, G.S. Gurdial, C.L. Liotta, and C.A. Eckert, "Intermolecular Interactions in Supercritical Fluid Cosolvent Effects," submitted to AIChE J. (1992).

Knutson, B.L.; Tomasko, D.L.; Eckert, C.A.; Petsche, I.B.; and Debenedetti, P.G., "Local Density Augmentation in Supercritical Solutions: A Comparison Between Fluorescence Spectroscopy and Molecular Dynamics," ACS Symp. Ser., 1992, in press.

Mayer, M.M.; Howell, W.J.; Tomasko, D.L.; and Eckert, C.A., "Solid-Liquid Equilibria in the Systems: Thianthiene + Phenanthrene, Salicylic Acid + Phenenathrene, and 3Hydroxybenzoic Acid + Phenanthrene," J. Chem. Eng. Data, 1990, 35, 446.

Tomasko, D.L., B.L. Knutson, J.M. Coppom, W. Windsor, B. West, and C.A. Eckert, "Spectroscopic Investigation of Alcohol-Solute Interactions in Supercritical Carbon Dioxide," submitted to ACS Symp. Ser. (1991). 


\section{REFERENCES}

Bartle, K. D., A. A. Clifford, and S. A. Jafar, "Relationship between Retention of a Solid Solute in Liquid and Supercritical Fluid Chromatography and its Solubility in the Mobile Phase," J. Chem. Soc. Faraday Trans., 1990a, 86, 855.

Bartle, K. D., A. A. Clifford, and S. A. Jafar, "Measurement of Solubility in Supercritical Fluids Using Chromatographic Retention: the Solubility of Fluorene, Phenanthrene, and Pyrene in Carbon Dioxide," J. Chem. Eng. Data, 1990b, 35, 355.

Berger, T. A. and J. F. Deye, "Composition and Density Effects Using Methanol/Carbon Dioxide in Packed Column Supercritical Fluid Chromatography," Anal. Chem., 1990, 62, 1181.

I rennecke, J.F., D.L. Tomasko, J. Peshkin, and C.A. Eckert, "Fluorescence Spectroscopy Studies of Dilute Supercritical Solutions," Ind. Chem. Eng. Res., 1990, 29, 1682.

Brennecke, J. F.; Eckert, C. A., "Phase Equilibria for Supercritical Fluid Process Design," AlChE J. 1989a, 35(9), 1409.

B. ennecke, J. F.; Eckert, C. A., "Fluorescence Spectroscopy Studies of Intermolecular Interactions in Supercritical Fluids," ACS Symp. Series 406., K. P. Johnston and J. M. L. Penninger, eds. 1989b, 14.

Brnnecke, J. F.; Tomasko, D.L.; Eckert, C. A., "Pyrene Excimer and Naphthalene/Triethylamine Exciplex Formation in Supercritical Fluid Solutions," J. Phys. Chem., 1990, $94,7692$.

Brown, B. O., A. J. Kishbaugh, and M. E. Paulaitis, "Experimental Determination of Enhancement Factors from Supercritical-Fluid Chromatography," Fluid Phase Equil., 36, 247 (1987).

Crow, J. A., and J. P. Foley, "Formic Acid Modified Carbon Dioxide as a Mobile Phase in Capillary Supercritical Fluid Chromatography," J. Microcol. Sep., 1991, 3, 47.

Cochran, H. D.; Lee, L. L., "Solvation Structure in Supercritical Fluid Mixtures Based on Molecular Distribution Functions," ACS Symp. Series 406, K. P. Johnston and J. M. L. Penninger, eds., 1989, 27.

Debenedetti, P.G., "Clustering in Dilute, Binary Supercritical Mixtures: A Fluctuation Analysis," Chem. Eng. Sci., 1987, 42, 2203.

Dobbs, J. M., J. M. Wong, and K. P. Johnston, "Nonpolar Co-Solvents for Solubility Enhancement in Supercritical Fluid Carbon Dioxide," J. Chem. Eng. Data, 1986, 31 , 
303 (1986).

Dobbs, J. M. and K. P. Johnston, "Selectivities in Pure and Mixed Supercritical Fluid Solvents," Ind. Eng. Chem. Res., 1987, 26, 1476.

Dobbs, J. M., J. M. Wong, R. J. Lahiere, and K. P. Johnston, "Modification of Supercritical Fluid Phase Behavior Using Polar Cosolvents," Ind. Eng. Chem. Res., 1987,26, 56.

Dolezalek, F., Z. Phys. Chem., 1908, 64, 727.

Dong, D. C.; Winnik, M. A., "The Py Scale of Solvent Polarities. Solvant Effects on the Vibronic Fine Structure of Pyrene Fluorescence and Empirical Correlations with $\mathrm{E}_{\mathrm{T}}$ and Y Values," Photochem. Photobiol. 1982, 35, 17.

Dong, D. C.; Winnik, M. A., "The Py Scale of Solvent Polarities," Can. J. Chem. 1984, 62, 2560.

Eckert, C.A., M.M. McNiel, B.A. Scott, and L.A. Halas, "NMR Measurements of Chemical Theory Equilibrium Constants for Hydrogen-Bonded Solutions," AIChE J., 1986a, 32, 820.

Eckert, C.A., D.H. Ziger, K.P. Johnston, and S. Kim, "Solute Partial Molal Volumes in Supercritical Fluids," J. Phys. Chem., 1986b, 90, 2738.

Ekart, M.P., K.I. Bennett, S.M. Ekart, G.S. Gurdial, C.L. Liotta, and C.A. Eckert, "Intermolecular Interactions in Supercritical Fluid Cosolvent Effects," submitted to AIChE J. (1992).

Foster, N.R., University of New South Wales, personal communication, 1990.

Fowler, L., W.N. Trump, and C.E. Vogler, "Vapor Pressure of Naphthalene. New Measurements between $40^{\circ}$ and $180^{\circ}$ C," J. Chem. Eng. Data, 13, 209 (1968).

Heidemann, R. A. and J. M. Prausnitz, "A van der Waals-Type Equation of State for Fluids with Associating Molecules," Proc. Natl. Acad. Sci. USA, 1976, 73, 1773.

Howell, W.J., C.T. Lira, and C. A. Eckert, "A Linear Chemical-Physical Theory Model for Liquid Metal Solution Thermodynamics," AlChE J., 1988, 34, 1477.

Hu, Y., E. Azevedo, D. Lüdecke, and J. Prausnitz, "Thermodynamics of Associated Solutions: Henry's Constants for Nonpolar Solutes in Water," Fl. Phase Equil., 1984, 17, 303. 
Hudson, G.H. and I.C. McCoubrey, Trans. Faraday Soc., 1960, 56, 761.

Hultgren, R., P. D. Desai, D.T. Hawkins, M. Gleiser, and K.K. Kelley, Selected Values of the Thermodynamic Properties of Binary Alloys, American Society for Metals, Metals Park, OH (1973).

Huppert, D., Kolodney, E., Gutman, M., and Nachliel, E., "Effect of Water Activity on the Rate of Proton Dissociation," J. Am. Chem. Soc., 1982, 104, 6949.

Ikonomou, G.D. and M.D. Donohue, "Extension of the Associated Perturbed Anisotropic Chain Theory to Mixtures with More Than One Associating Component," Fl. Phase Equil., $1988,39,129$.

Ireland, J.F., Wyatt, P.A.H., "Acid-Base Properties of Electronically Excited States of Organic Molecules," Adv. Phys. Org. Chem., 1976, 12, 131.

Johnston, K.P.; Peck, D.G.; Kim, S., "Modeling Supercritical Mixtures: How Predictive is it?," Ind. Eng. Chem. Res. 1989a, 28, 1115.

Johnston, K.P.; Kim, S.; Combes, J., "Spectroscopic Determination of Solvent Strength and Structure in Supercritical Fluid Mixtures: A Review," ACS Symp. Series 406, K. P. Johnston and J. M. L. Penninger, eds., 1989b, 52.

Kajimoto, O., M. Futakami, T. Kobayashi, and K. Yamasaki, "Charge-Transfer-State Formation in Supercritical Fluids: (N,N-Dimethylamino)benzonitrile in $\mathrm{CF}_{3} \mathrm{H}$," J. Phys. Chem., 1988, 92, 1347.

Kalyanasundaram, K.; Thomas, J. K., "Environmental Effects on Vibronic Band Intensities in Pyrene Monomer Fluorescence and Their Application in Studies of Micellar Systems," J. Amer. Chem. Soc. 1977, 99, 2039.

Karachewski, A.M., M.M. McNiel, and C.A. Eckert, "A Study of Hydrogen Bonding in Alcohol Solutions Using NMR Spectroscopy," Ind. Eng. Chem. Res., 1989, 28, 315.

Kim, S.; Johnston, K. P., "Clustering in Supercritical Fluid Mixtures," AIChE J. 1987a, 33(10), 1603.

Kim, S.; Johnston, K. P., "Molecular Interactions in Dilute Supercritical Fluid Solutions," Ind. Eng. Chem. Res. 1987b, 26, 1206.

Kosal,E.; Holder, G.D., "Solubility of Anthracene and Phenathrene Mixtures in Supercritical Carbon Dioxide," J. Chem. Eng, Data, 1987, 32(2),148. 
Lee, J., Griffin, R.D., and Robinson, G.W., "2-Naphthol: A Simple Example of Proton Transfer Effected by Water Structure," J. Chem. Phys., 1985, 82(11), 4920.

Lee, J., Robinson, G.W., Webb, S.P., Phillips, L.A., and Clark, J.H., "Hydration Dynamics of Protons from Photon Initiated Acids," J. Am. Chem. Soc., 1986, 108, 6538.

Levy, J. M. and W. M. Ritchey, "The Effects of Modifiers in Supercritical Fluid Chromatography," J. High Res. Chrom. Chrom. Comm., 1985, 8, 503.

Lumb, M. D. Luminescence Spectroscopy, Academic Press, N.Y., 1978.

Morris, R.W. and E.A. Turek, "Optimal Temperature-Dependent Parameters for the RedlichKwong Equation of State," ACS Symp. Ser., 1986, 300, 389.

Nakajima, A., "Solvent Effect on the Vibrational Structures of the Fluorescence and Absorption Spectra of Pyrene," Bull. Chem. Soc. Jap. 1971, 44, 3272.

Petsche, I.; Debenedetti, P. G., "Solute-Solvent Interactions in Infinitely Dilute Supercritical Mixtures: A Molecular Dynamics Investigation," J. Chem. Phys., 1989, 91(11), 7075.

Schmitt, W.J. and R.C. Reid, "Solubility of Monofunctional Organic Solids in Chemically Diverse Supercritical Fluids," J. Chem. Eng. Data, 1986, 31, 204.

Shim, J.-J. and K. P. Johnston, "Phase Equilibria, Partial Molar Enthalpies, and Partial Molar Volumes Determined by Supercritical Fluid Chromatography," J. Phys. Chem., 1991, $95,353$.

Soave, G., "Equilibrium Constants from a Modified Redlich-Kwong Equation of State," Chem. Eng. Sci., 1972, 27, 1197.

Tomasko, D.L., B.L. Knutson, J.M. Coppom, W. Windsor, B. West, and C.A. Eckert, "Spectroscopic Investigation of Alcohol-Solute Interactions in Supercritical Carbon Dioxide," submitted to ACS Symp. Ser. (1991).

Tsekhanskaya, Y.V., M.B. Iomtev, and E.V. Mushkina, "Solubility of Naphthalene in Ethylene and Carbon Dioxide under Pressure," Russ. J. Phys. Chem., 38, 11, 1964.

Tsutsumi, K., Shizuka, H., "Proton Transfer and Acidity Constant in the Excited State of Naphthols by Dynamic Analyses," Z. Phys. Chem., 1980, 122, 129.

Van, S.P.; Hammond, G.S.; "Amine Quenching of Aromatic and Fluorescence and Fluorescent Exciplexes," J. Am. Chem. Soc., 1978, 100(12), 3895. 
Wu, R.S.; Lee, L.L.; Cochran, H.D., "Structure of Dilute Supercritical Fluid Solutions: Clustering of Solvent and Solute Molecules and the Thermodynamic Effects," Ind. Eng.Chem. Res., 1990, 29, 977.

Yonker, C. R.; Smith, R. D., "Retention in Supercritical Fluid Chromatography: Influence of the Partial Molar Volume of the Solute in the Stationary Phase," J. Phys. Chem. 1988, $92,1664$.

Yonker, C.R.; Smith, R.D., "Sorption Isotherms of Mobile Phase Components in Capillary Supercriticical Fluid Chromatography," J. Chrom., 1990, 505, 139. 


\section{SOLUBILITY OF 5,6 DIMETHYLBENZIMIDAZOLE IN SCF BUTANE}

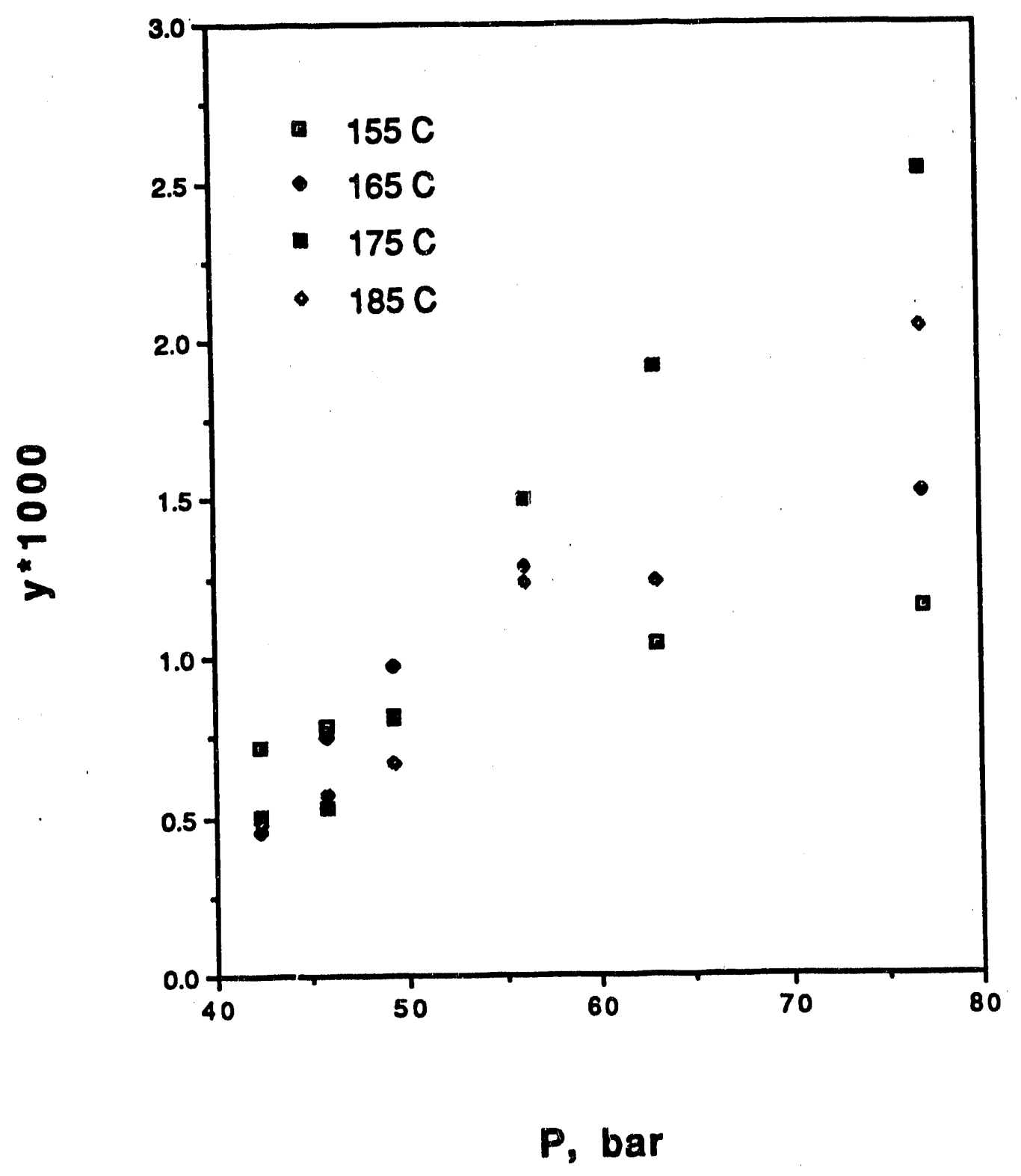

Figure 1. Solubility of 5,6 Dimethylbenzimidazole in SCF Butane 


\section{Solubility of Anthraquinone in SCF Butane}

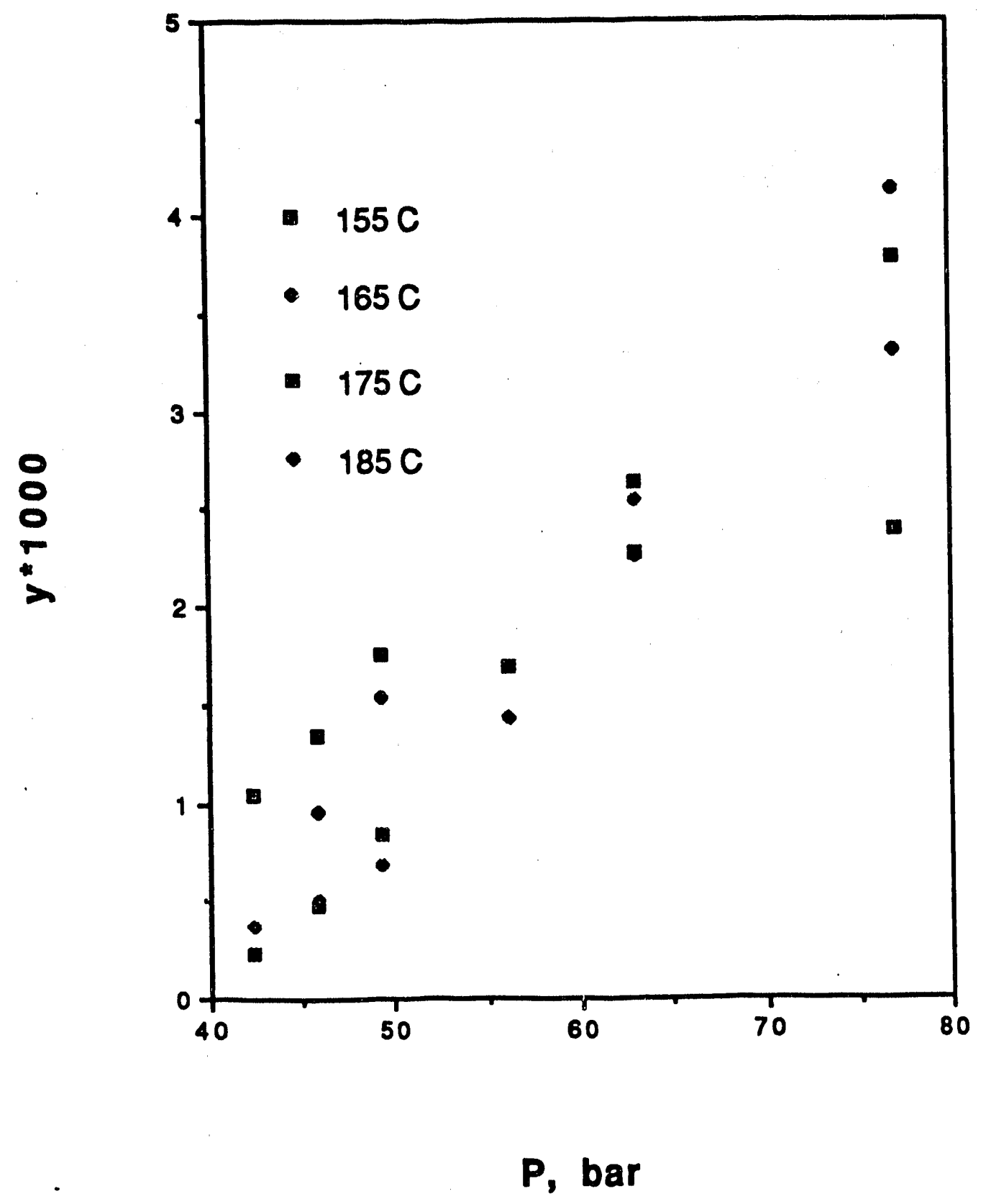

Figure :. Solubility of Anthraquinone in SCF Butane 


\section{Solubility of 9,10 Phenanthrenequinone In SCF Butane}

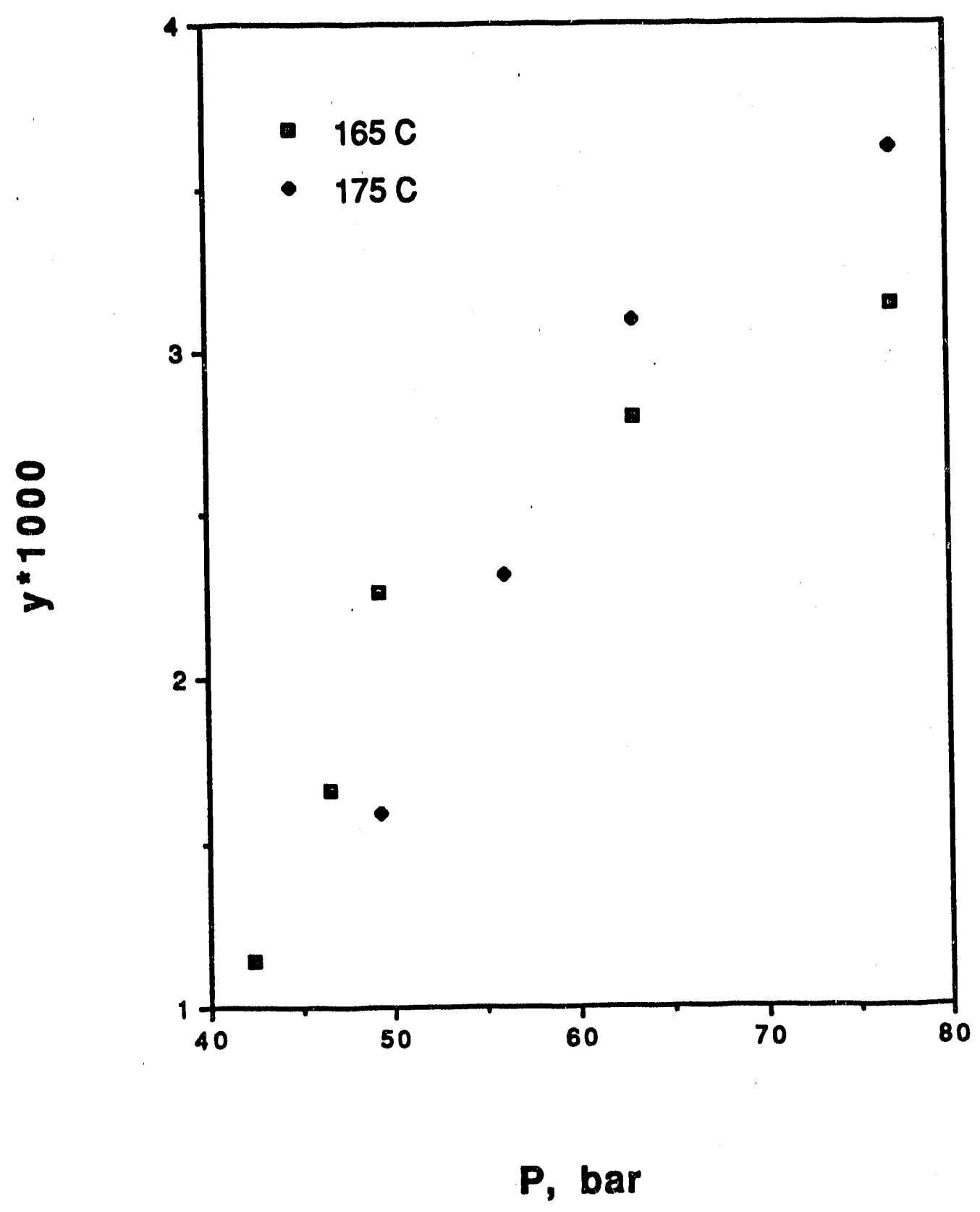

Figure 3. Solubility of 9,10 Phenanthrenequionoe in SCF Butane 


\section{UV ABSORPTION APPARATUS}

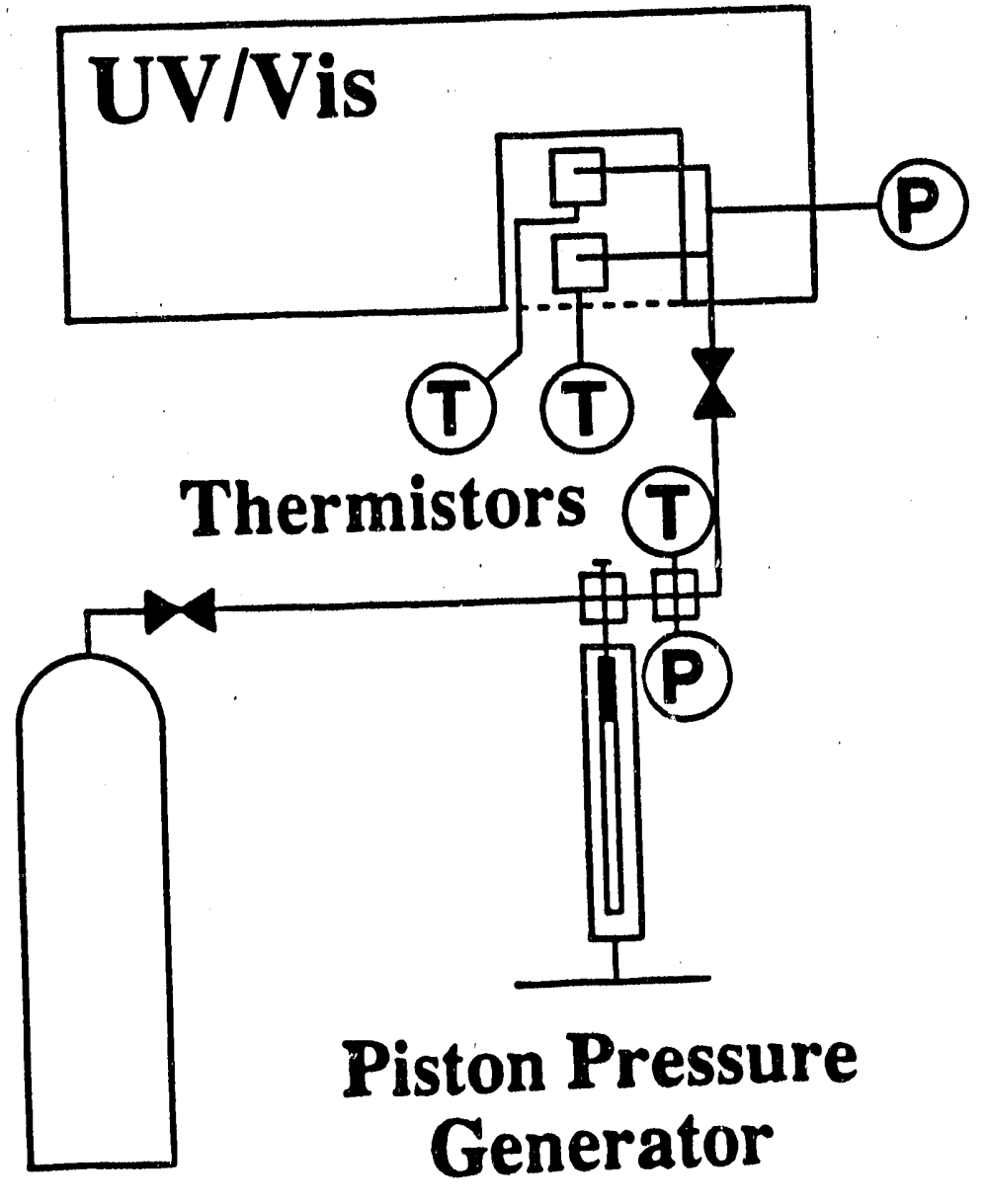

Figure 4. Schematic of UV Absorption Apparatus 


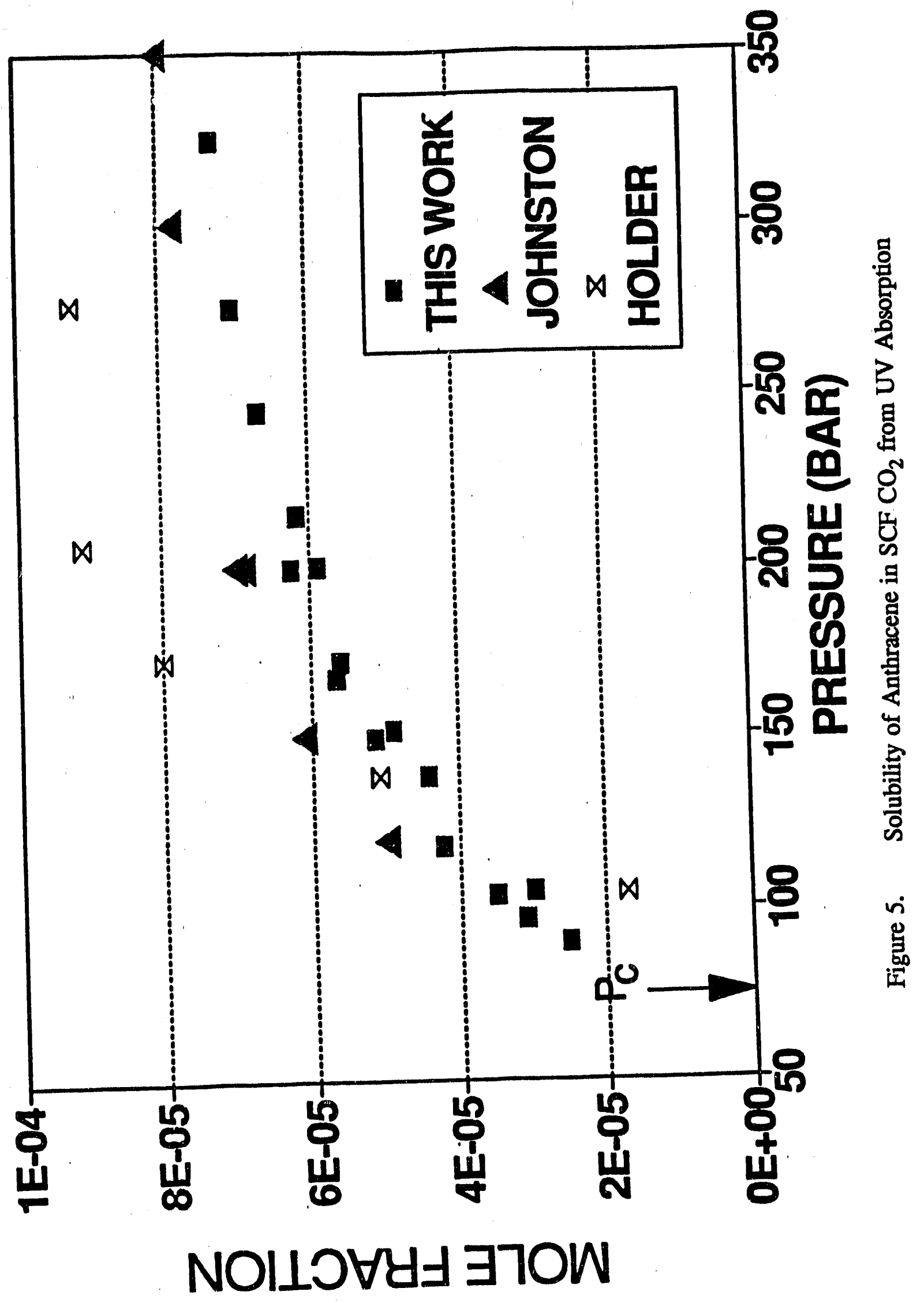




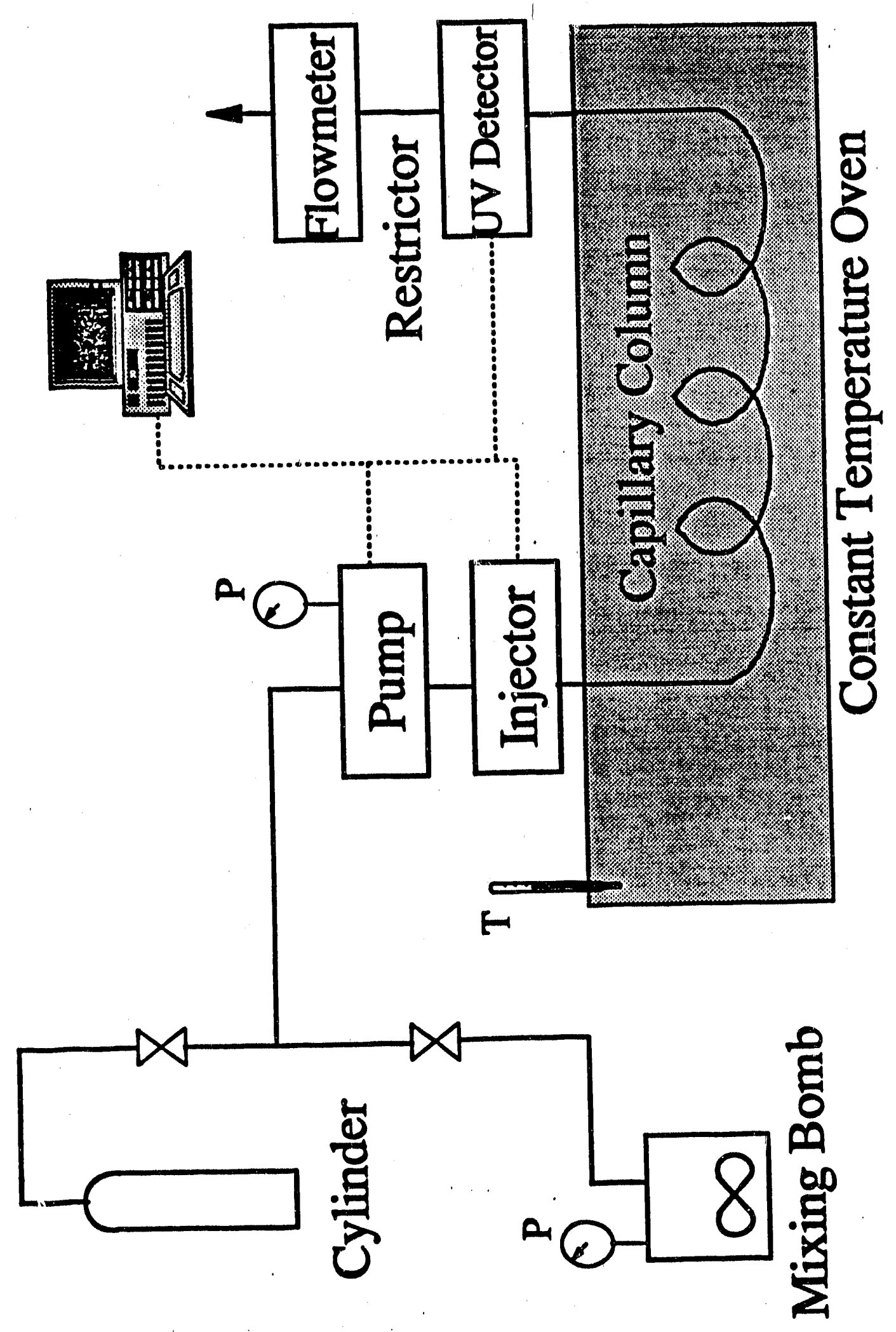

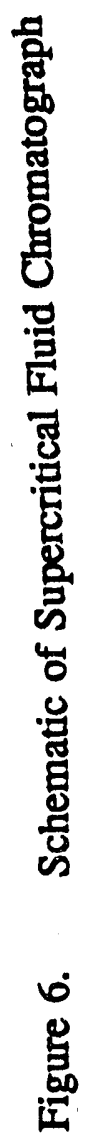




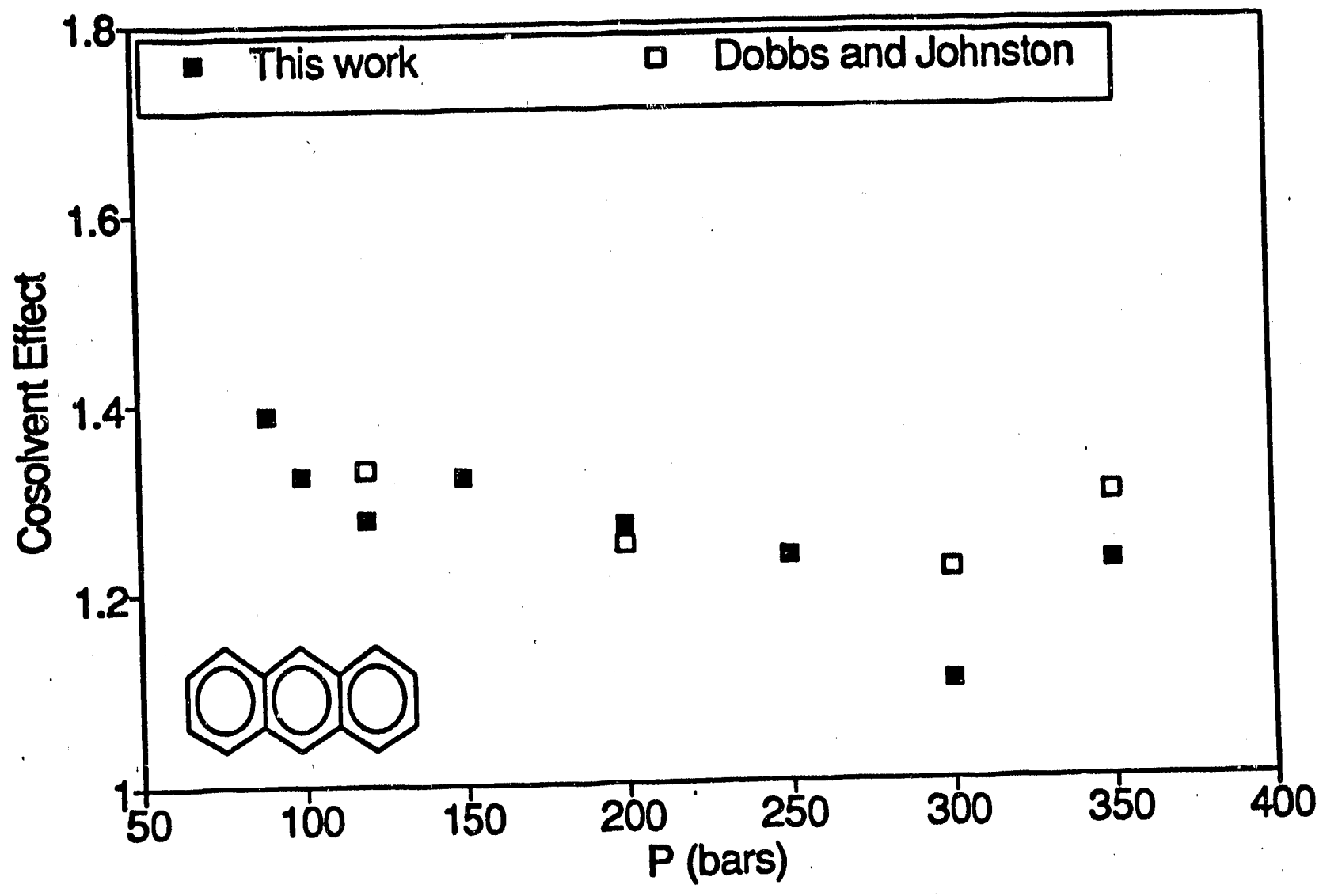

Figure 7. Cosolvent Effect for Anthracene in $\mathrm{CO}_{2}$ with $3.5 \mathrm{~mol} \%$ Methanol Relative to Pure $\mathrm{CO}_{2}$ at $35^{\circ} \mathrm{C}$ 


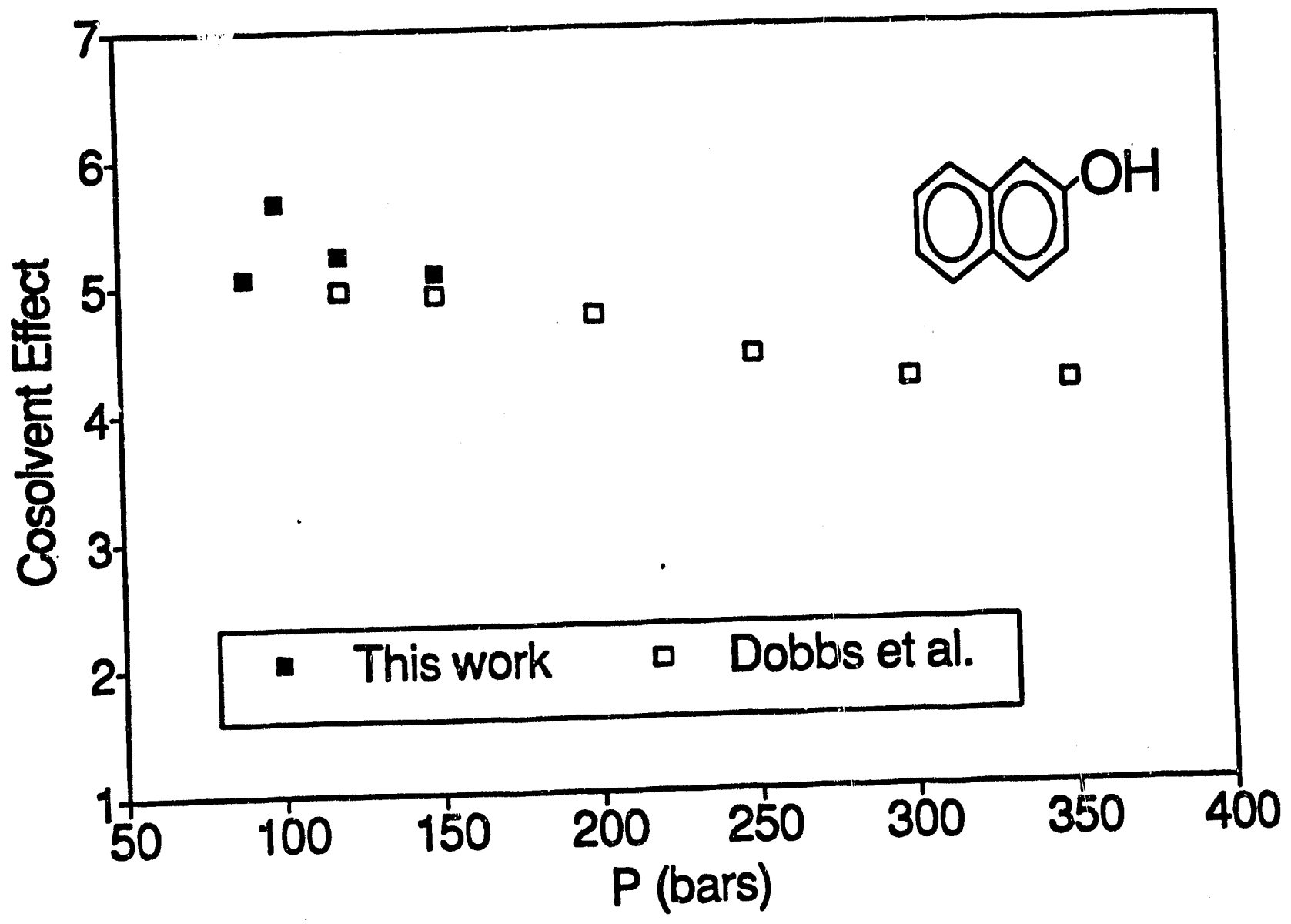

Figure 8. Cosolvent Effect for 2-Naphthol in $\mathrm{CO}_{2}$ with 3.5 mol\% Methanol Relative to Pure $\mathrm{CO}_{2}$ at $35^{\circ} \mathrm{C}$ 


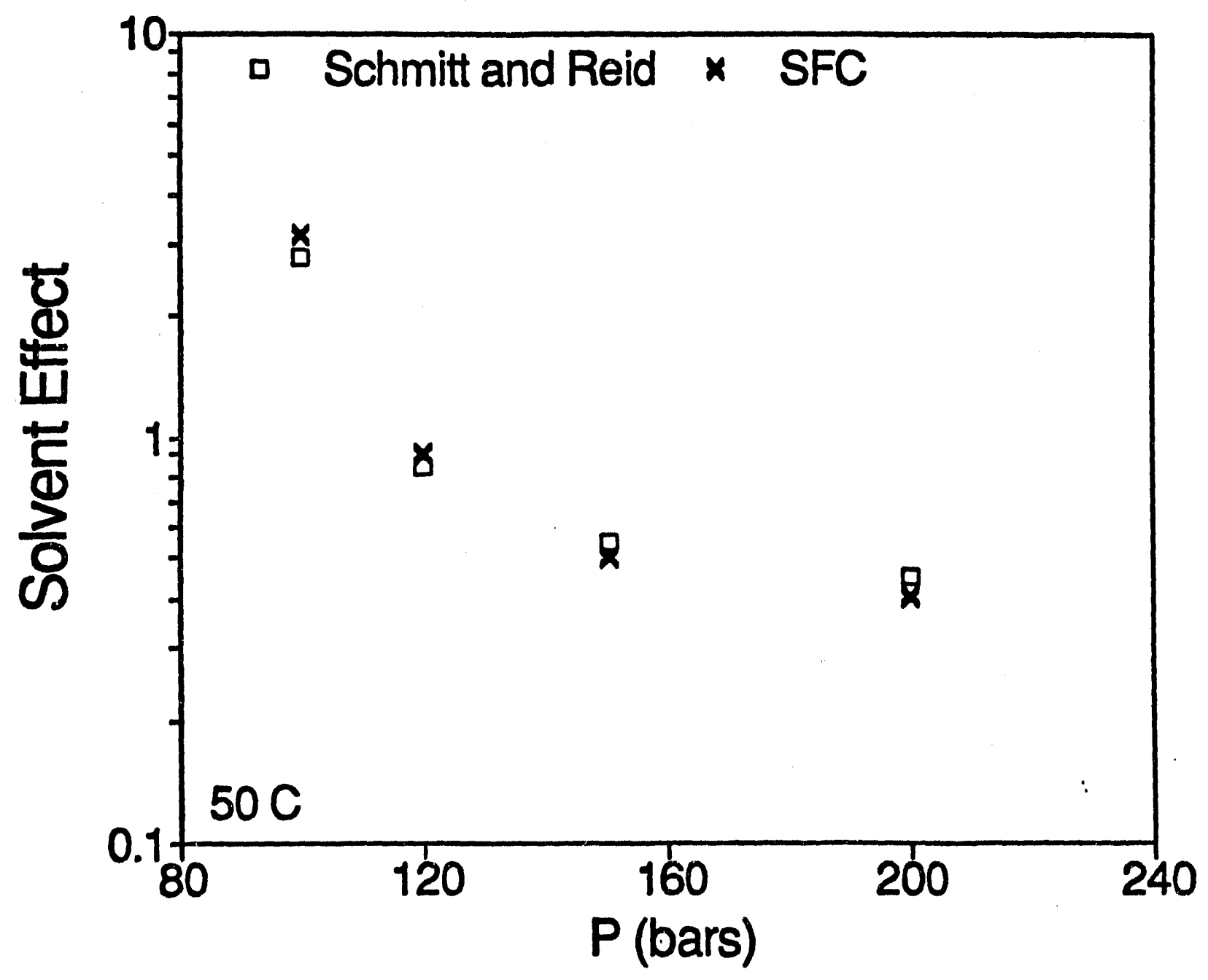

Figure 9. Solvent Effect for 2-Naphthol in Ethane Relative to Pure $\mathrm{CO}_{2}$ at $35^{\circ} \mathrm{C}$ 


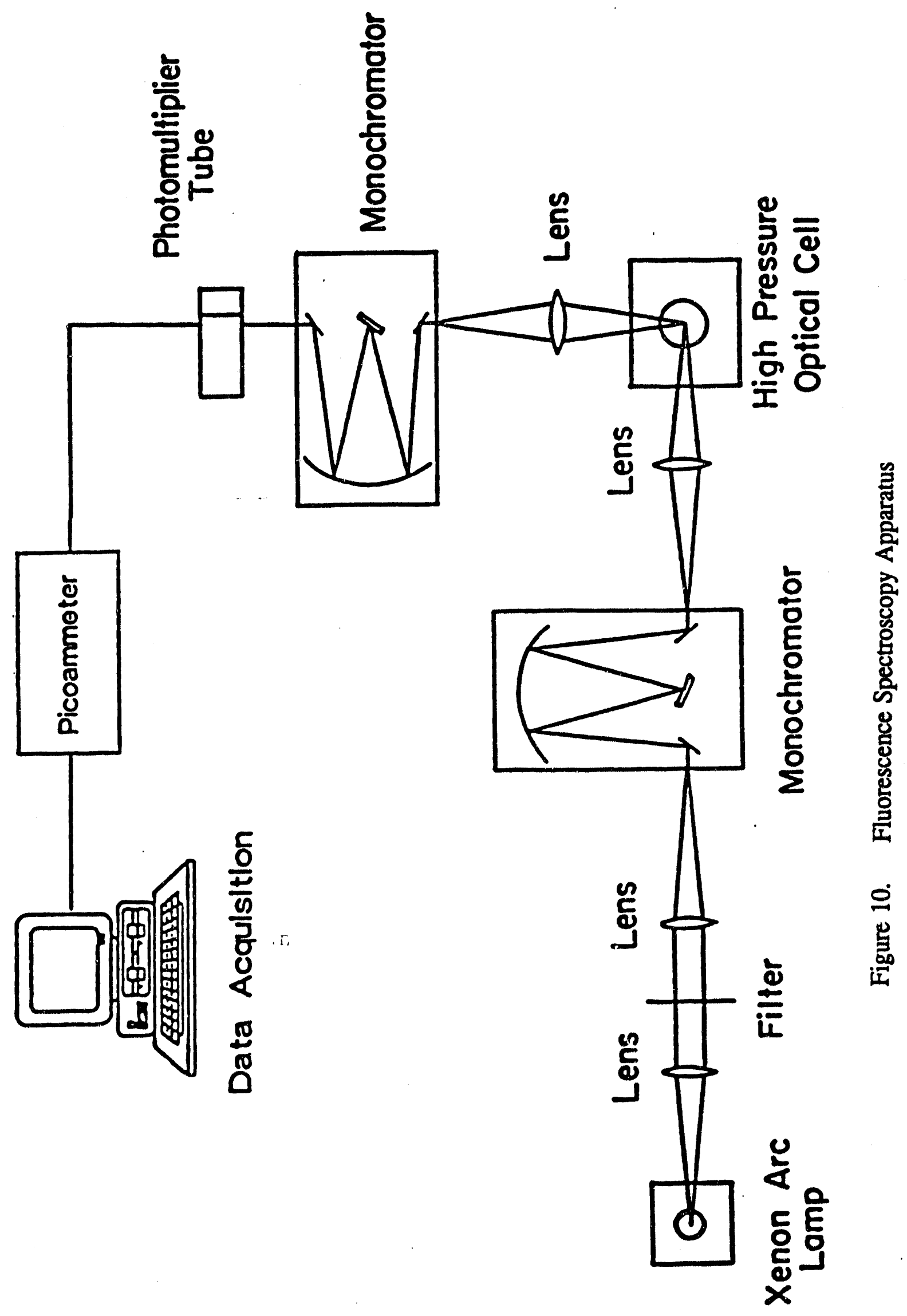




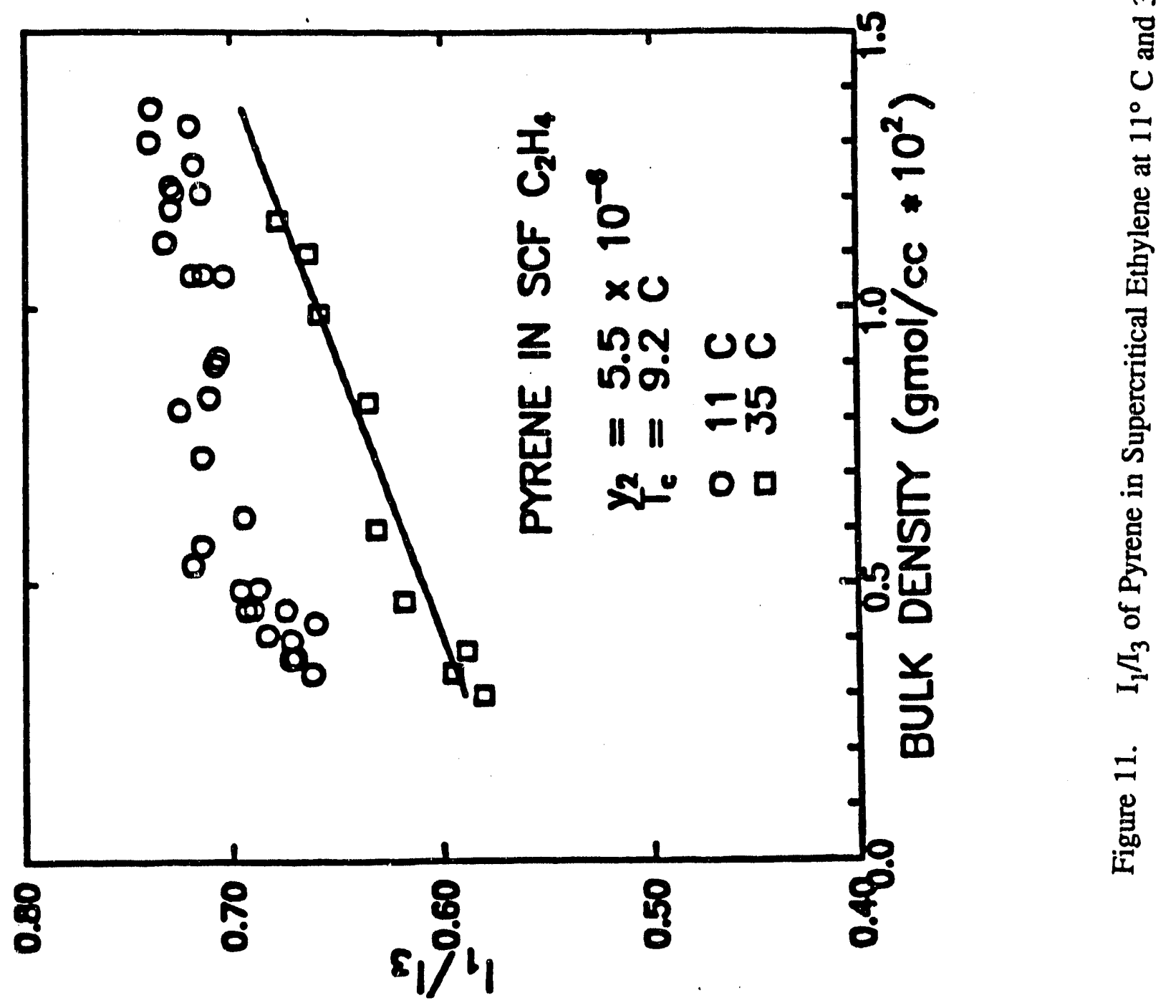




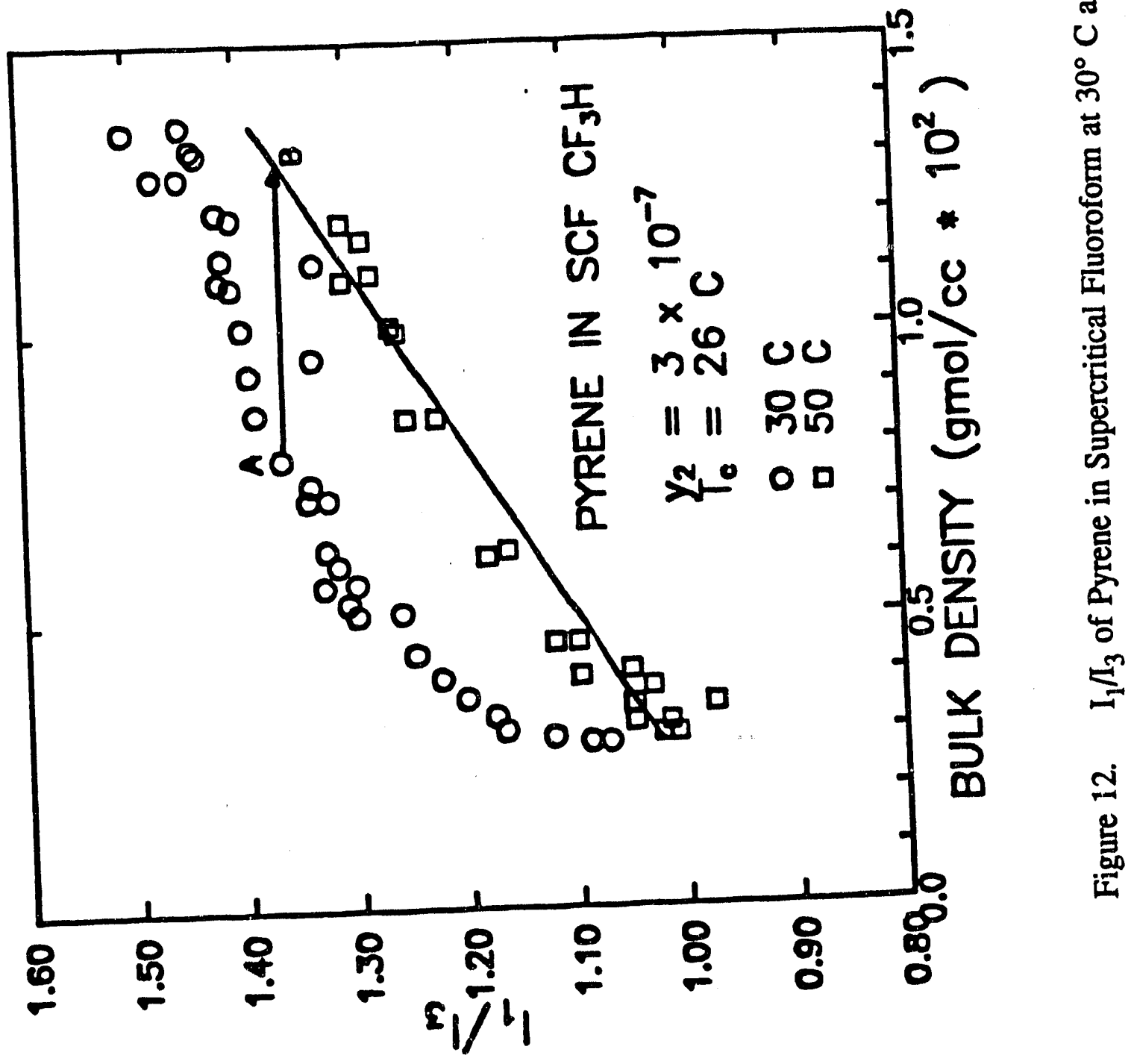




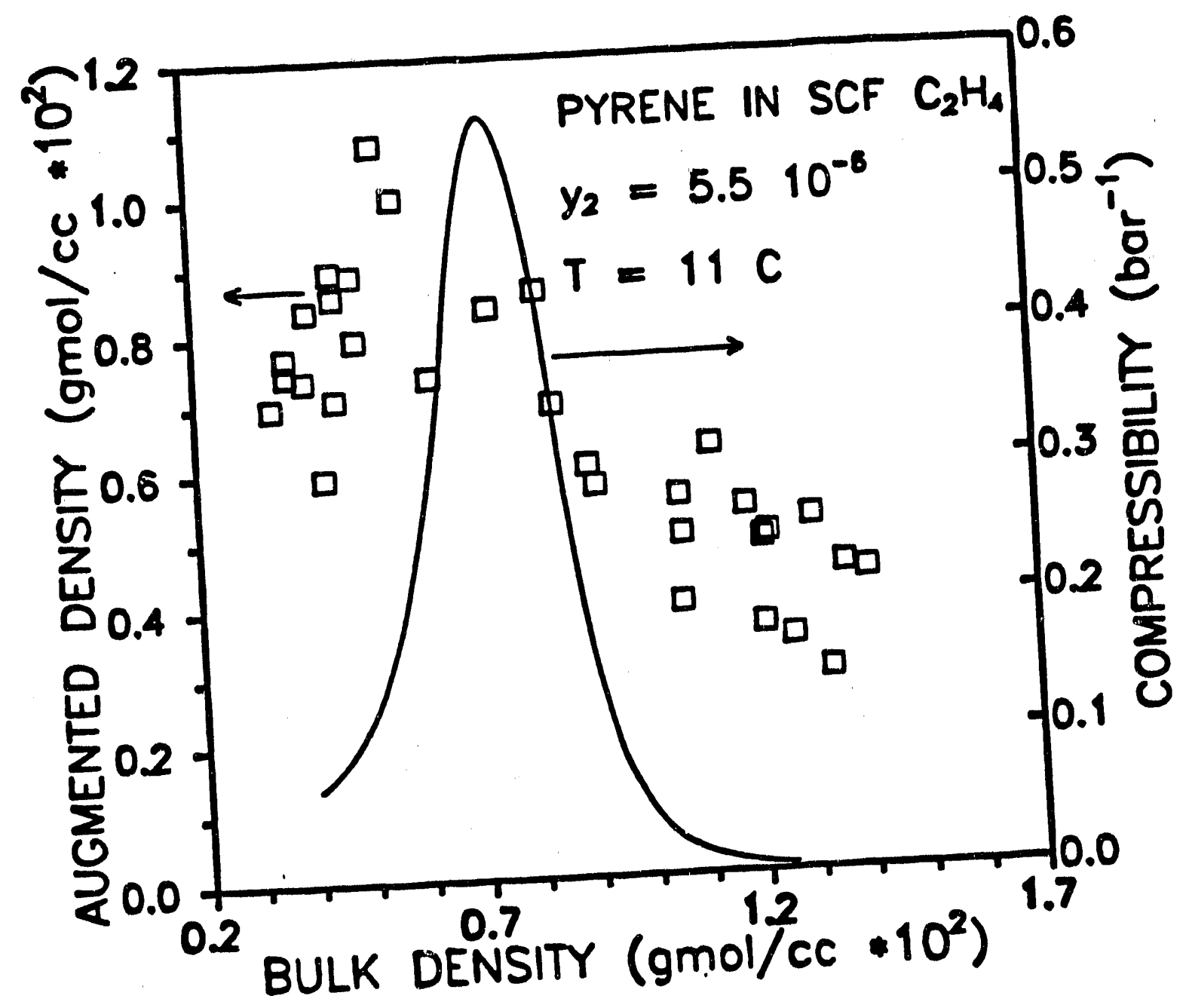

Figure 13. Augmented Denstiy around Pyrene in Supercritical Ethylene Superimposed on the Isothermal Compressibility of the Solvent 


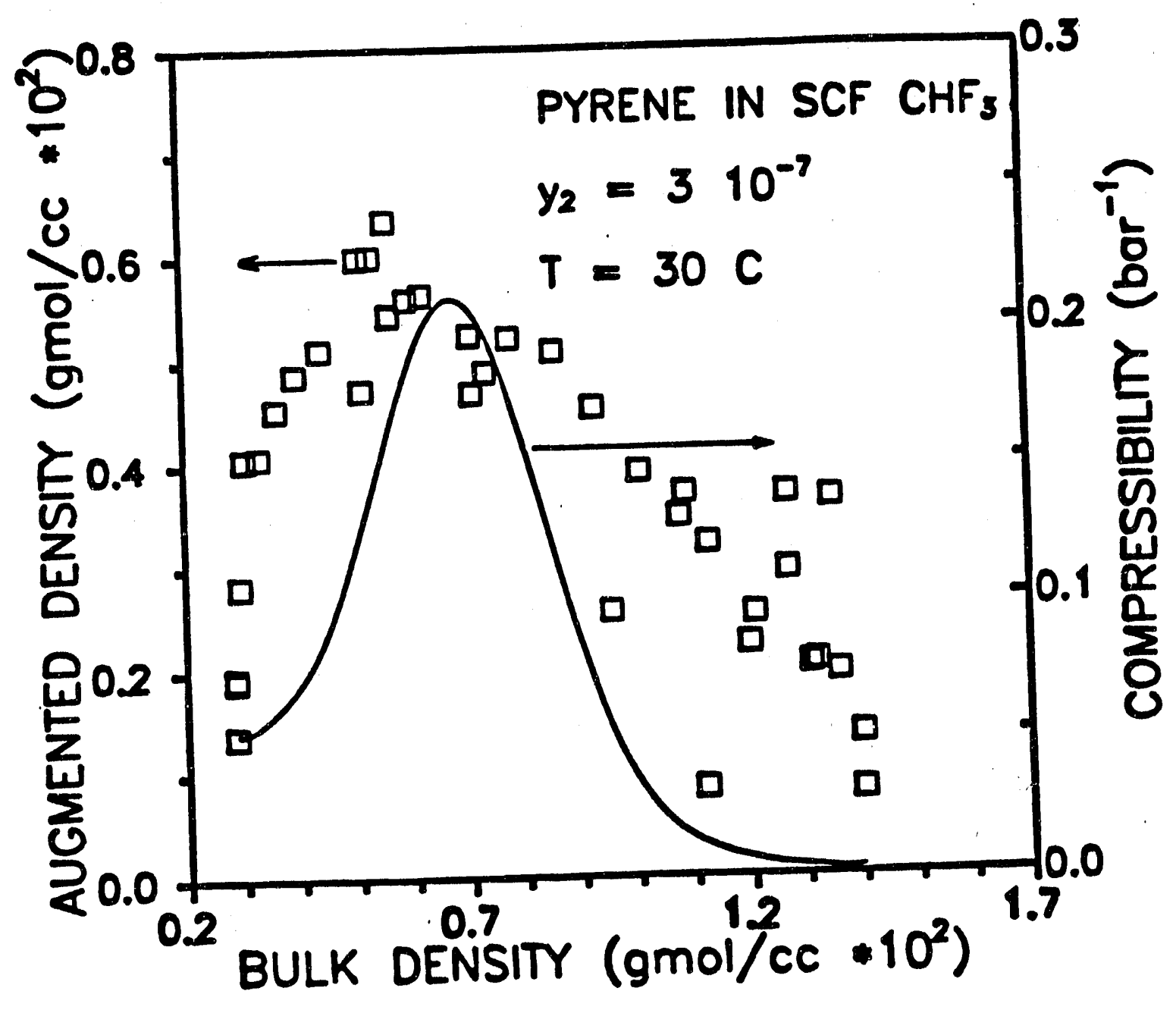

Figure 14. Augmented Denstiy around Pyrene in Supercritical Fluoroform Superimposed on the Isothermal Compressibility of the Solvent 


\section{PYRENE IN CO \\ $T_{R}=1.02$}

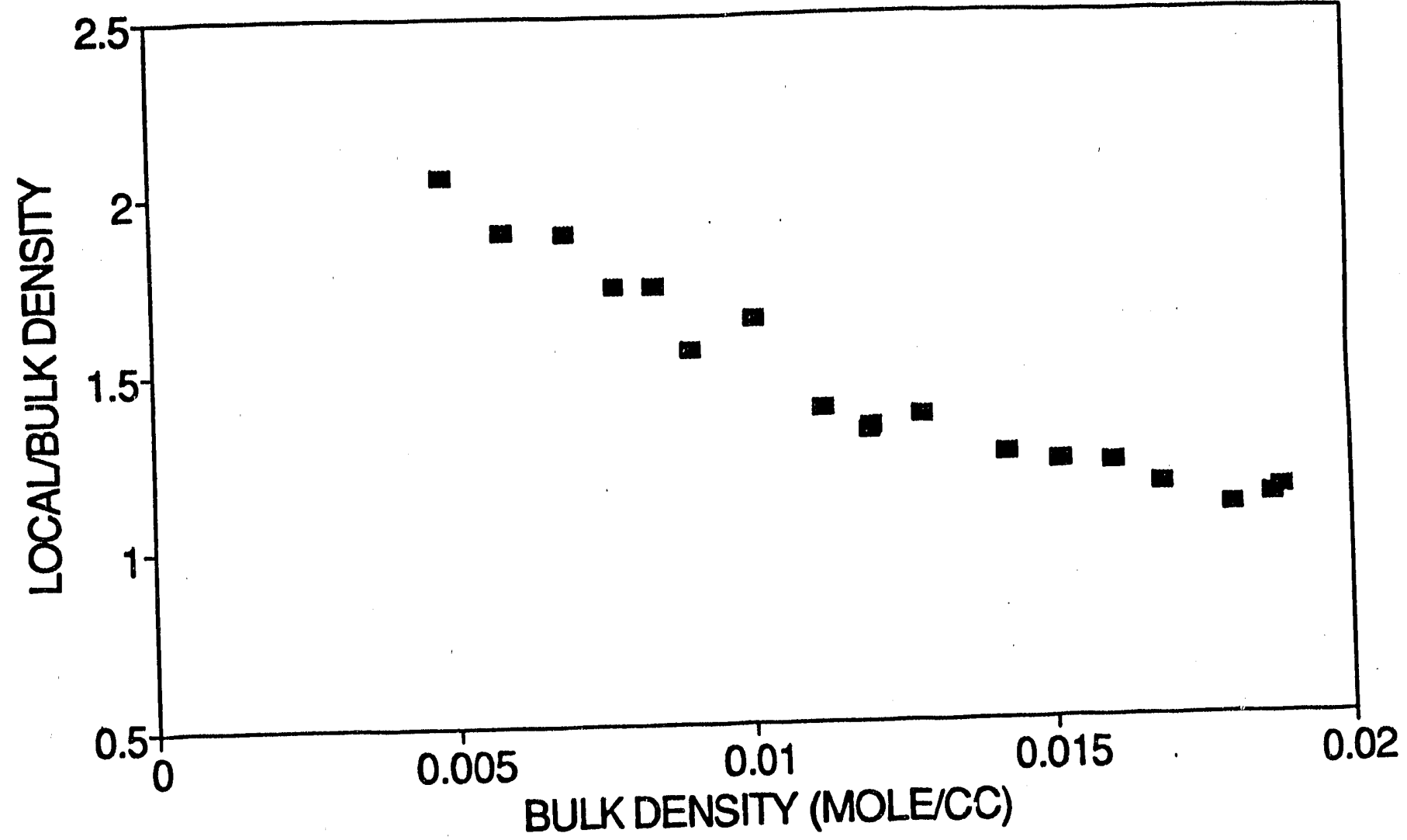

Figure 15. Local Density Enhancement as a Function of Bulk Density for Pyrene in SCF $\mathrm{CO}_{2}$ from Fluorescence Spectroscopy 
PYRENE IN CO

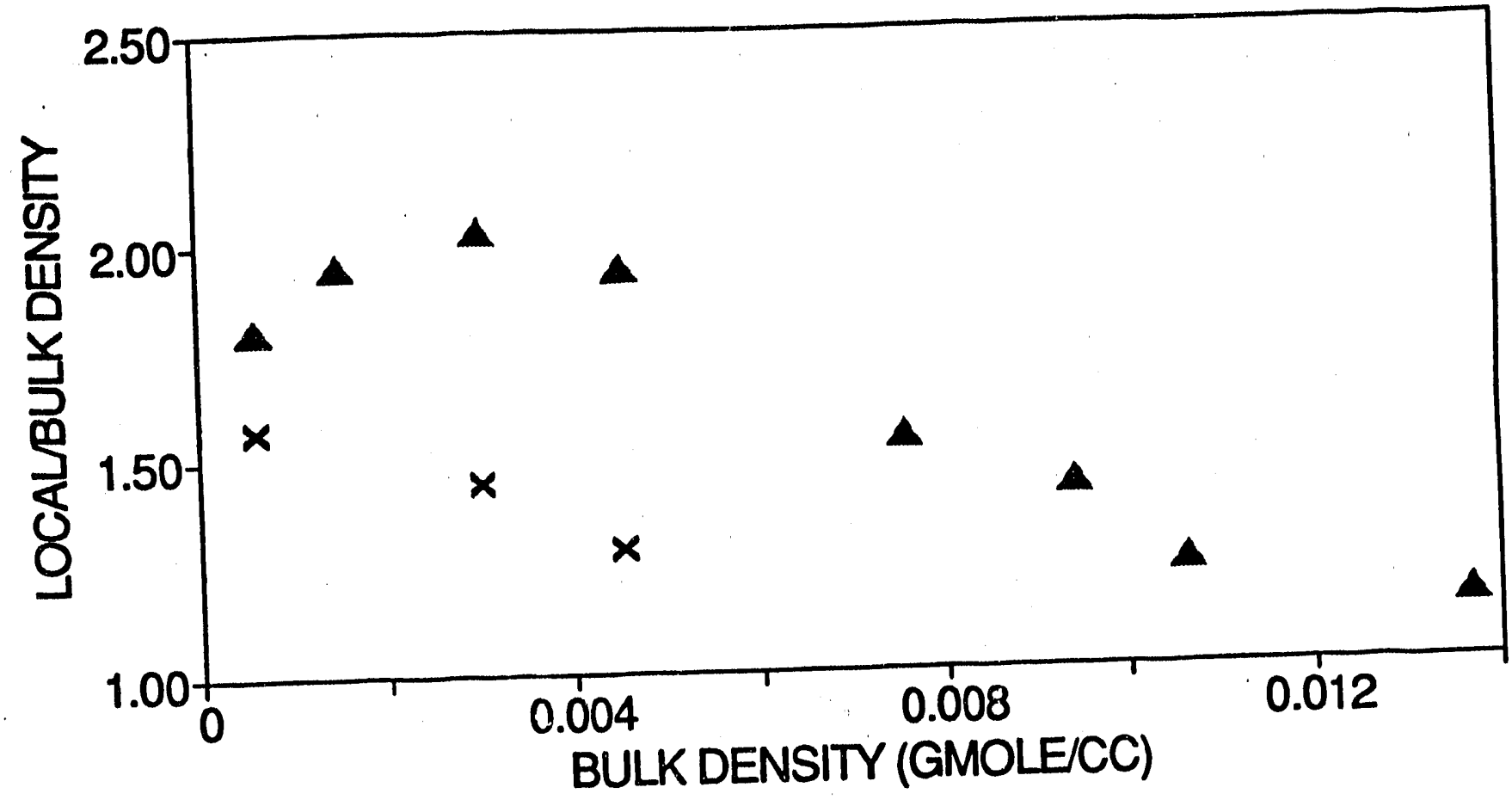

$$
T_{R}=1.02 \times T_{R}=1.14
$$

Figure 16. Local Density Enhancen ant as a Function of Bulk Density for Pyrene in SCF $\mathrm{CO}_{2}$ from Molecular Dy 


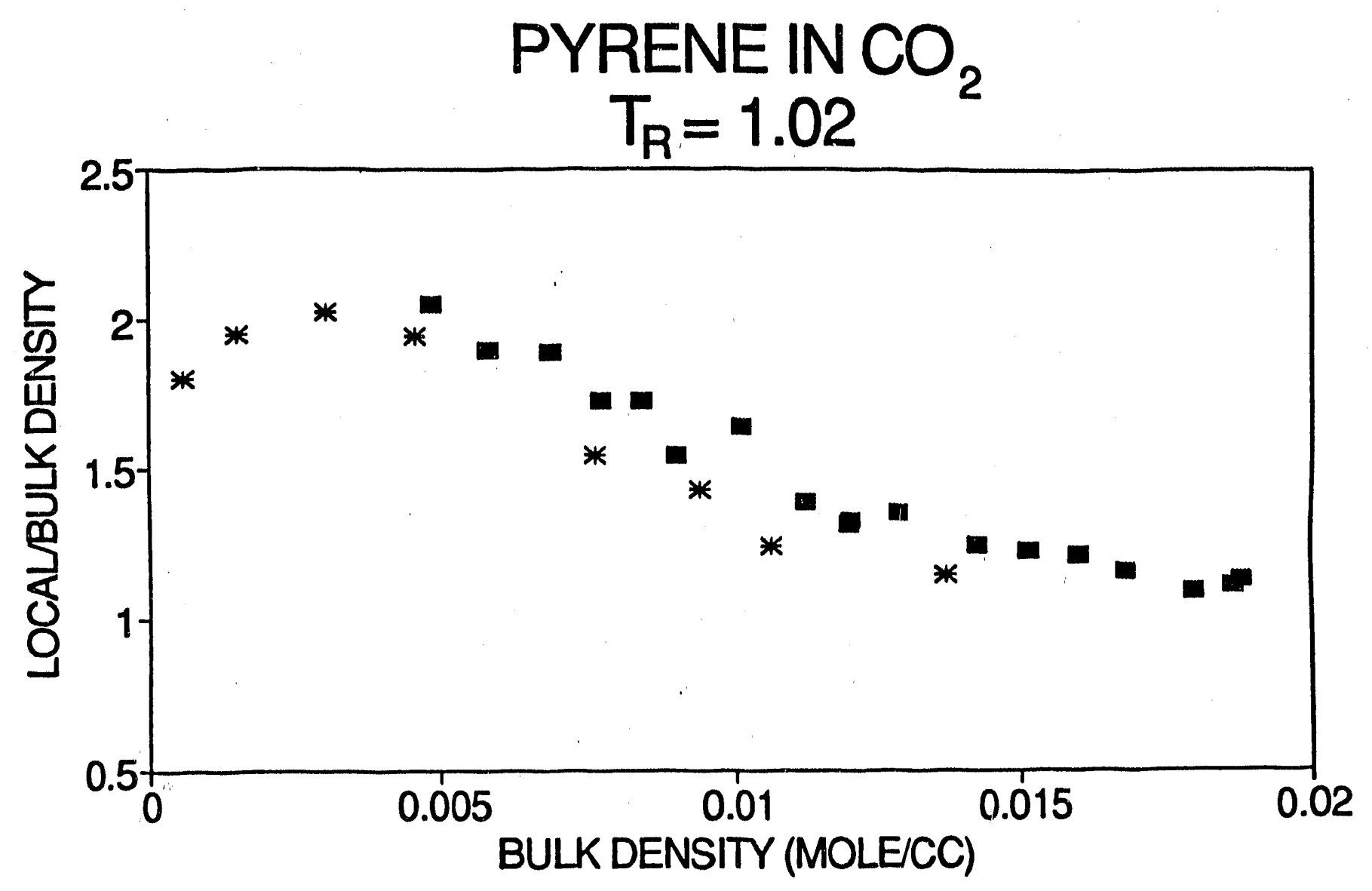

FLUORESCENCE * SIMULATION

Figure 17. Comparison of Local Density Enhancement as a Function of Bulk Density for Pyrene in SCF $\mathrm{CO}_{2}$ from Fluorescence Spectroscopy and Molecular Dynamics Simulations at $T_{R}=1.02$ 


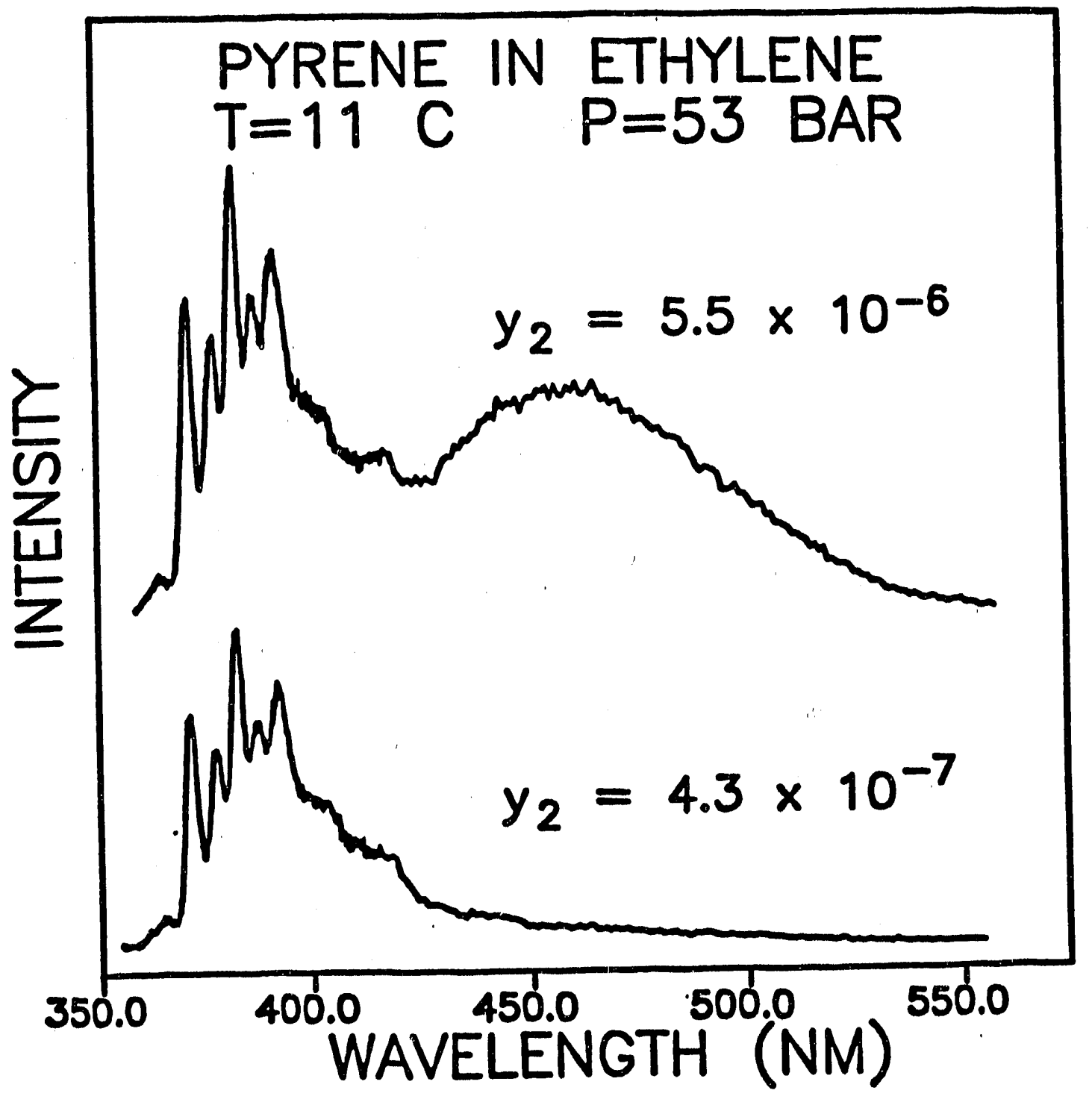

Figure 18. Fluorescence Spectra of Pyrene in SCF Ethylene at $11^{\circ} \mathrm{C}$ 


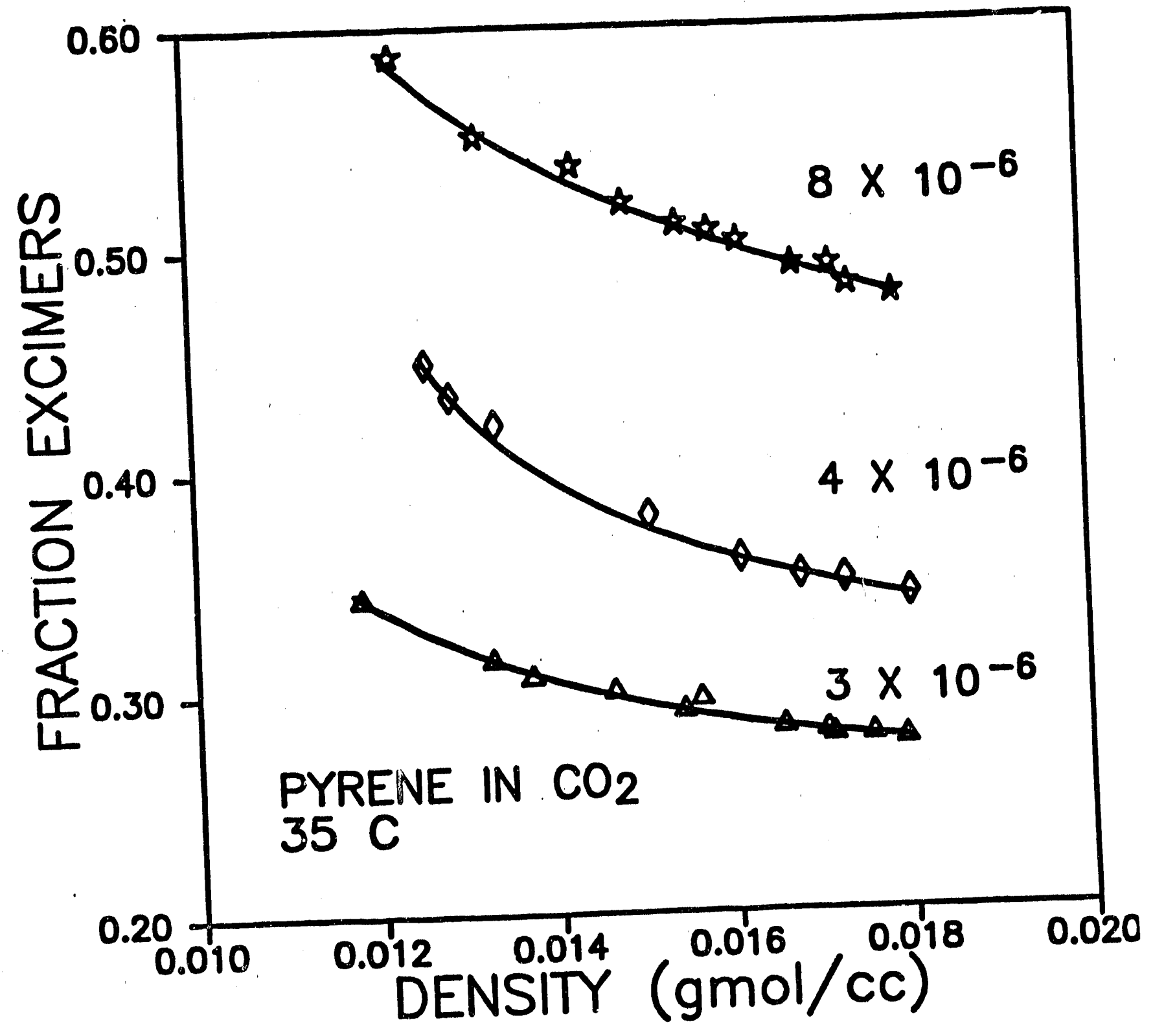

Figure 19. Fraction of Pyrene Molecules Fluorescing as Excimers in $\mathrm{SCF} \mathrm{CO}_{2}$ at $35^{\circ} \mathrm{C}$ 


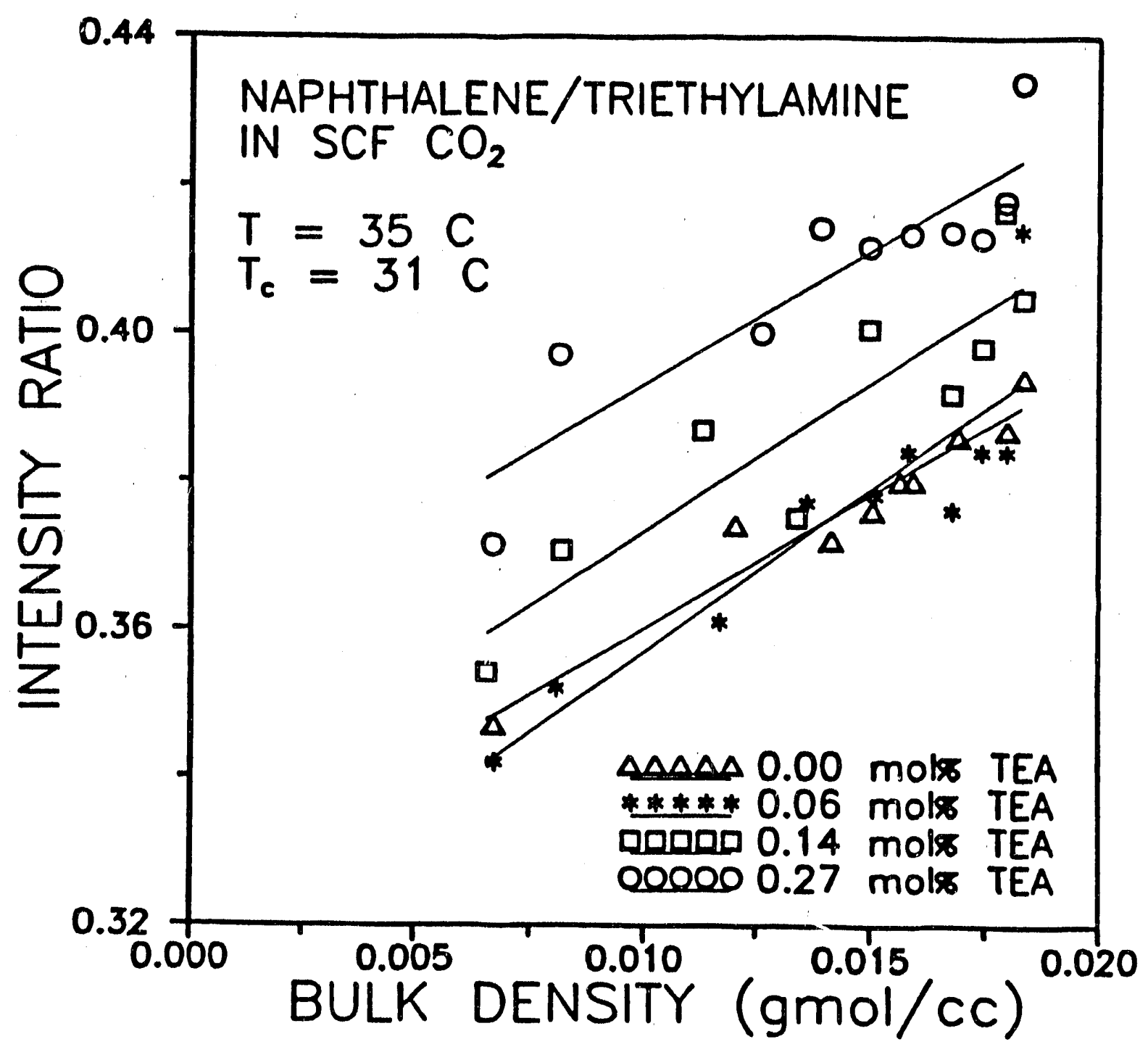

Figure 20. $\quad \mathrm{I}_{1} \mathrm{I}_{4}$ of Naphthalene in Supercritical $\mathrm{CO}_{2}$ at $35^{\circ} \mathrm{C}$ and Triethylamine Concentrations from 0.0 to 0.27 mole\% 


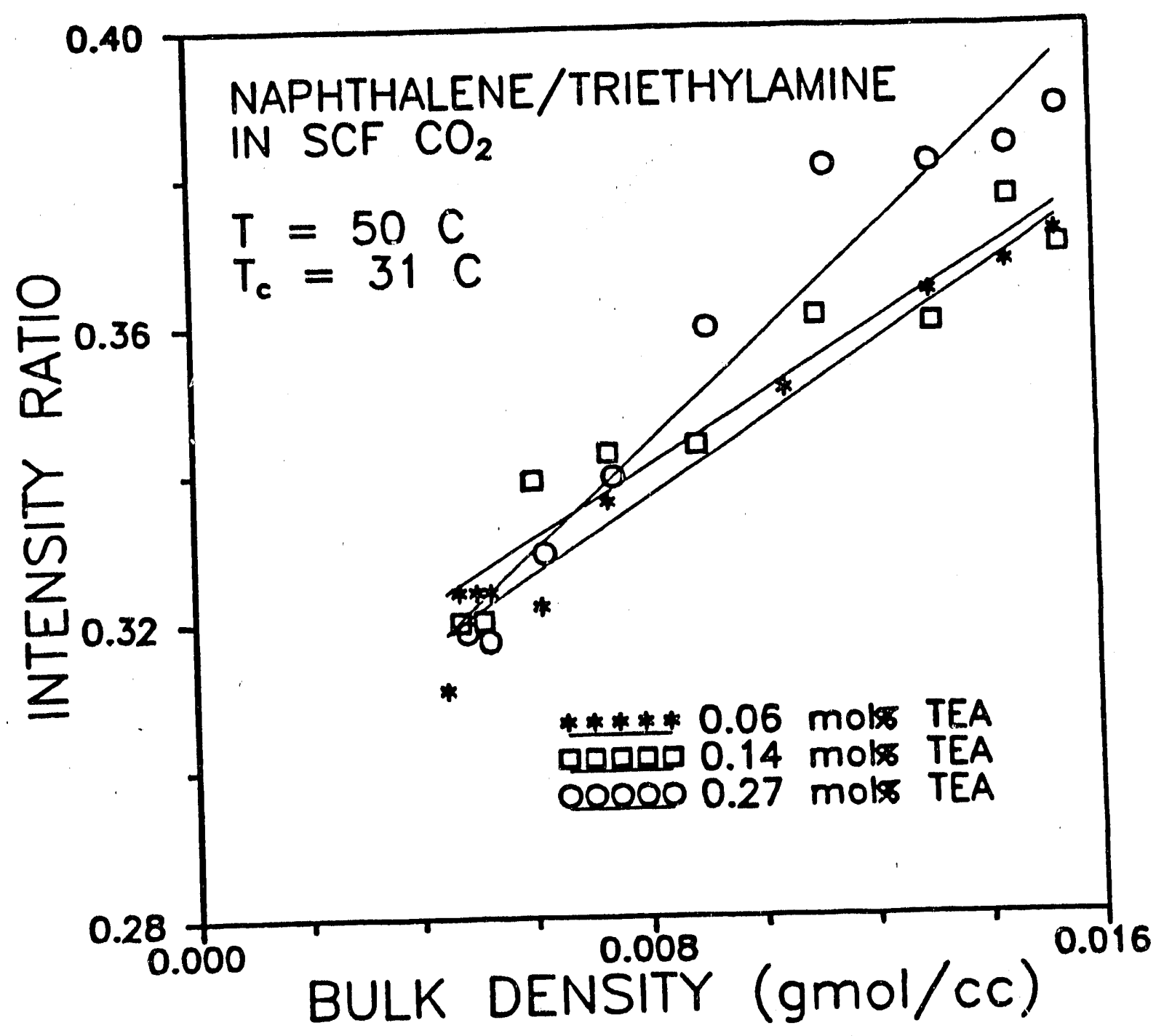
Figure 21. $\mathrm{I}_{1} \mathrm{I}_{4}$ of Naphthalene in Supercritical $\mathrm{CO}_{2}$ at $50^{\circ} \mathrm{C}$ and Triethylamine
Concentrations from 0.0 to 0.27 mole\% 


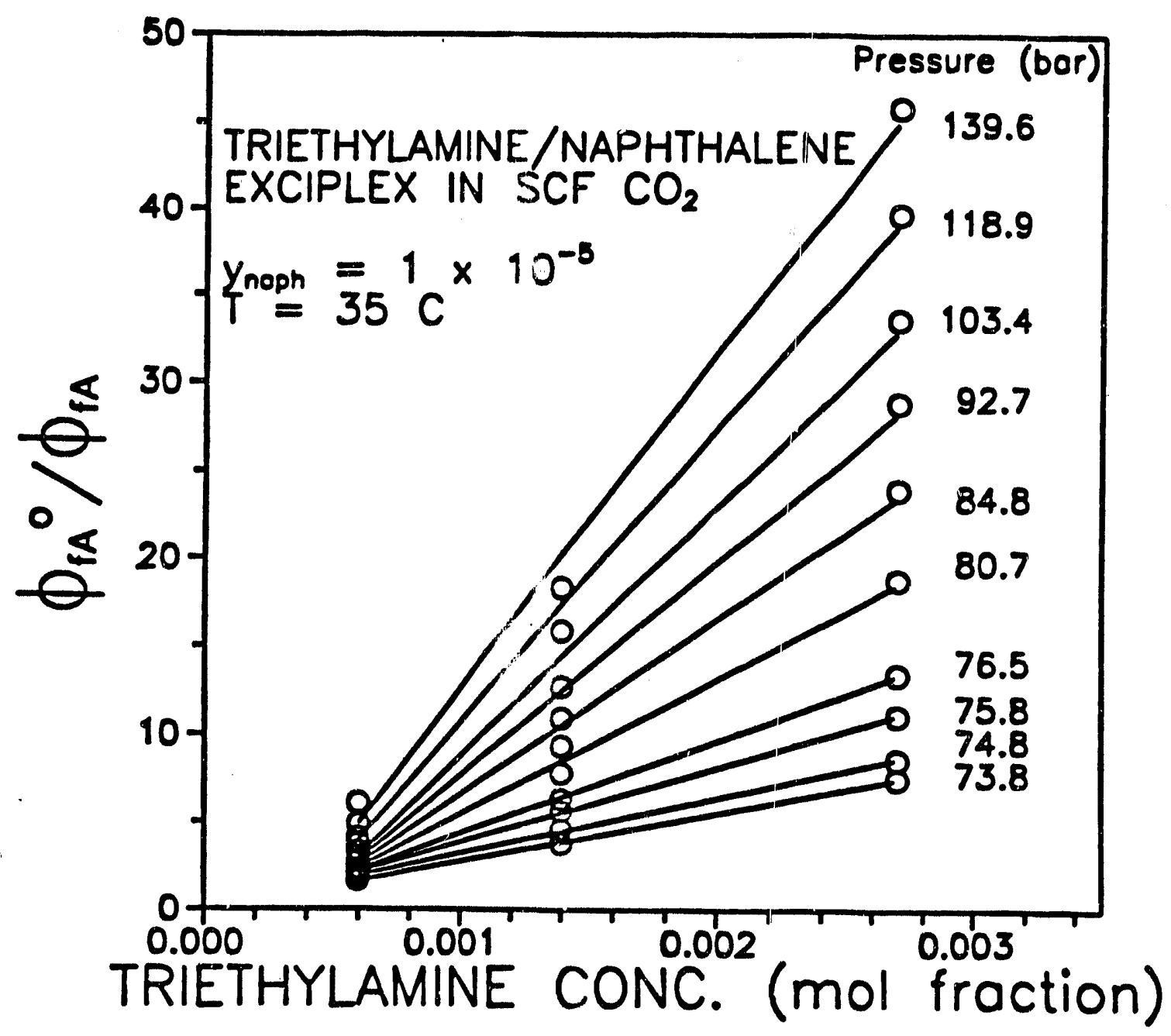

Figure 22. Ratio of Normal Fluorescence Area of Naphthalene without and with Triethylamine in Supercritical $\mathrm{CO}_{2}$ at $3{ }^{\prime} \mathrm{C}$ 


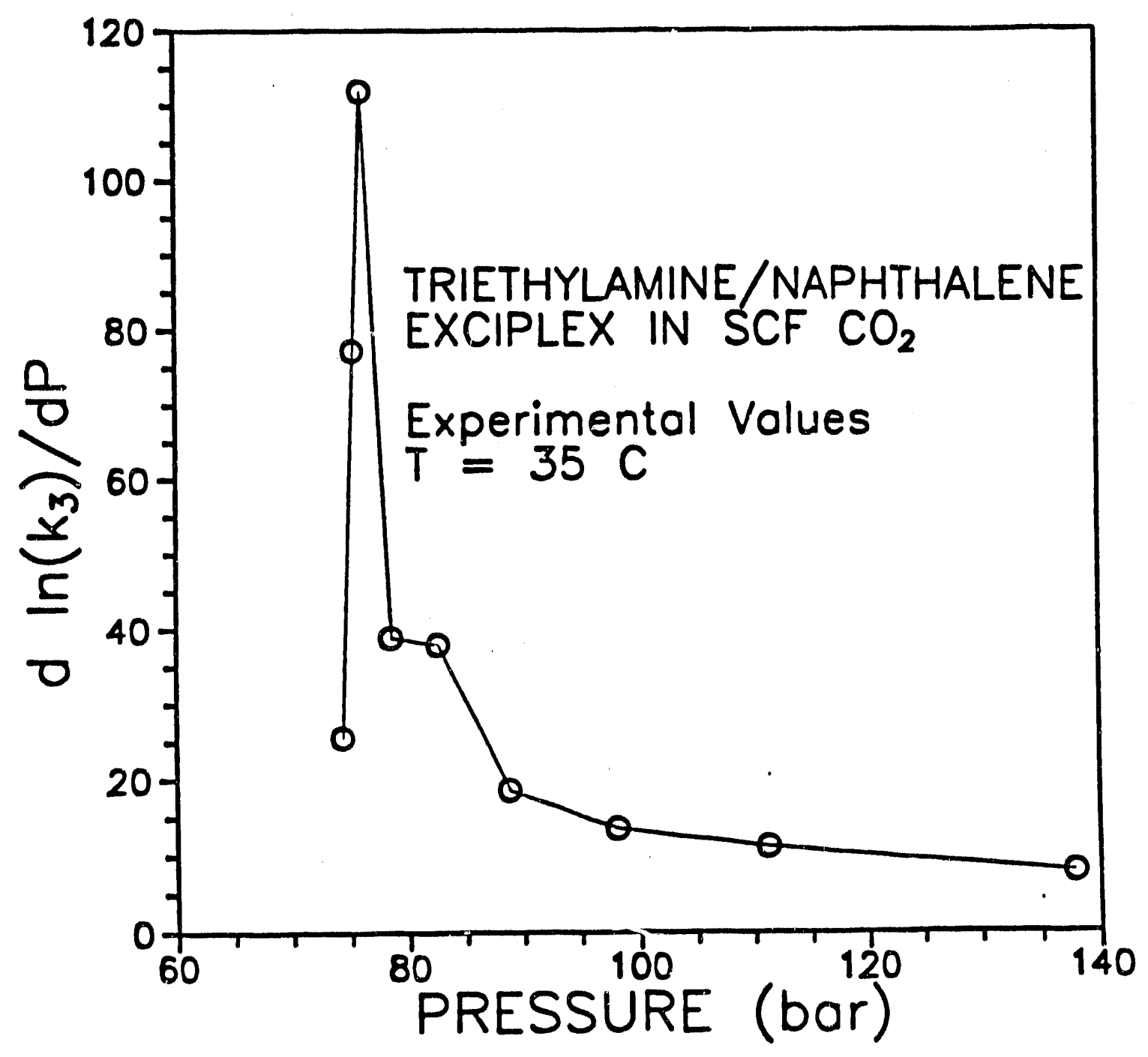
Figure 23. Pressure dependence of the rate constant for exciplex formation numericlally
determined from the slopes in Figure 22. The line connects experimental points. 


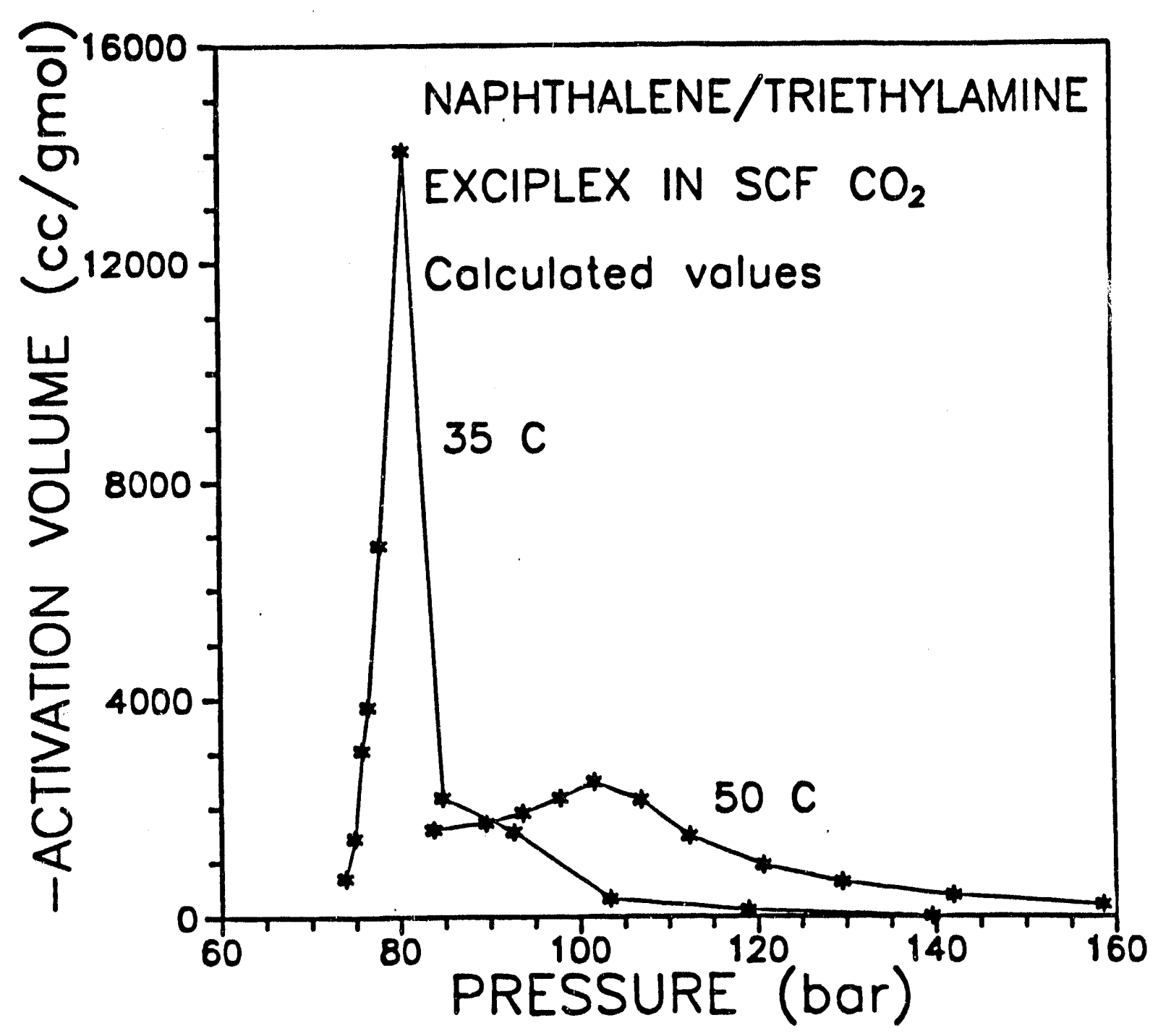

Figure 24. Negative of the activation volume calculated from the sum of the contributions from the Peng-Robinson equation of state. The line connects the points. 


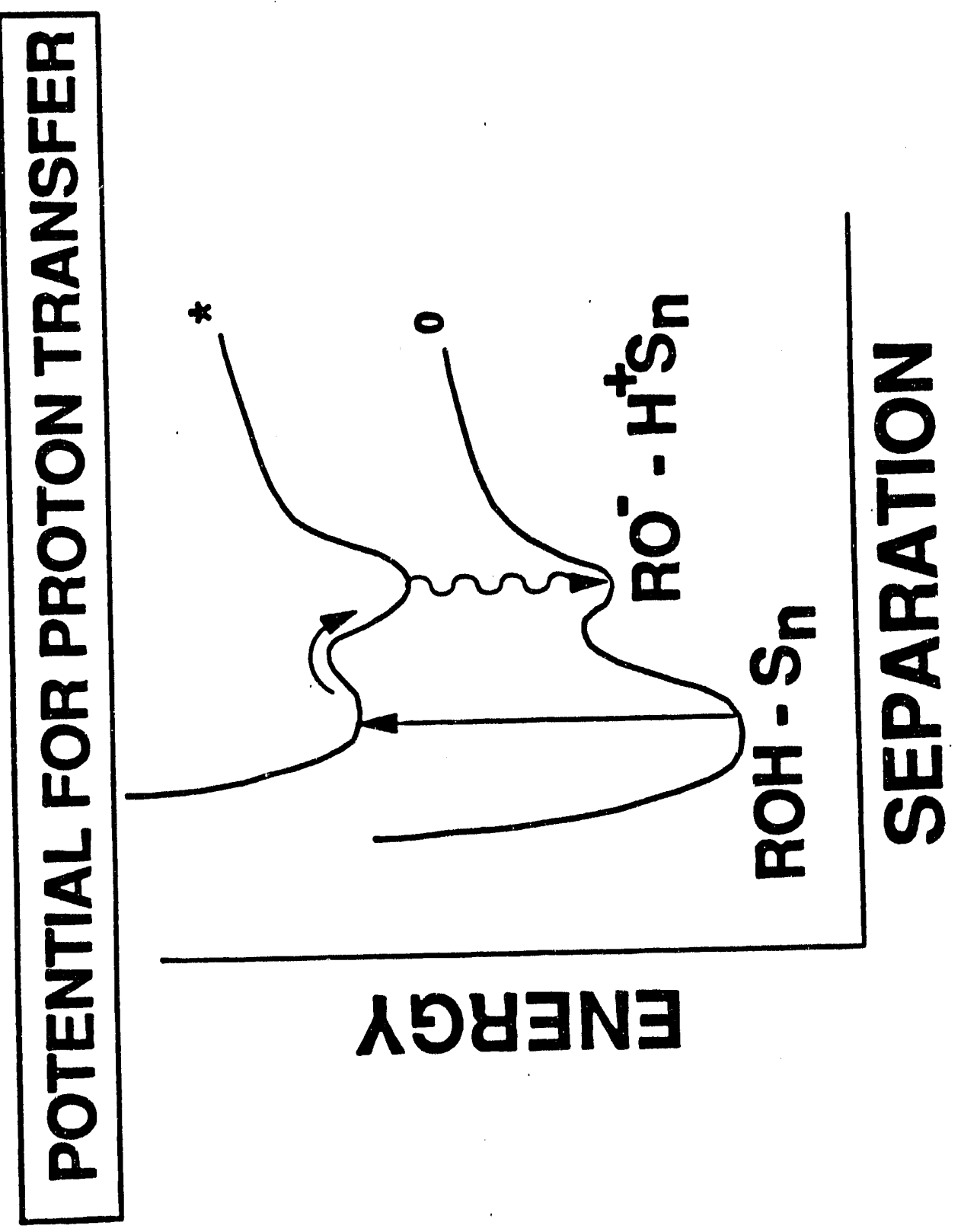

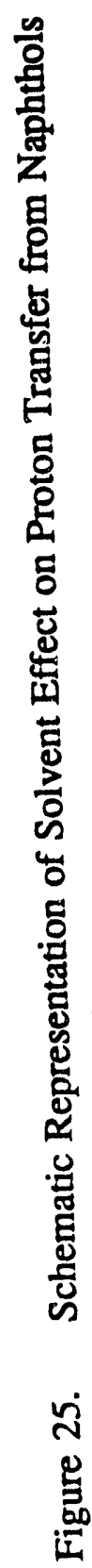




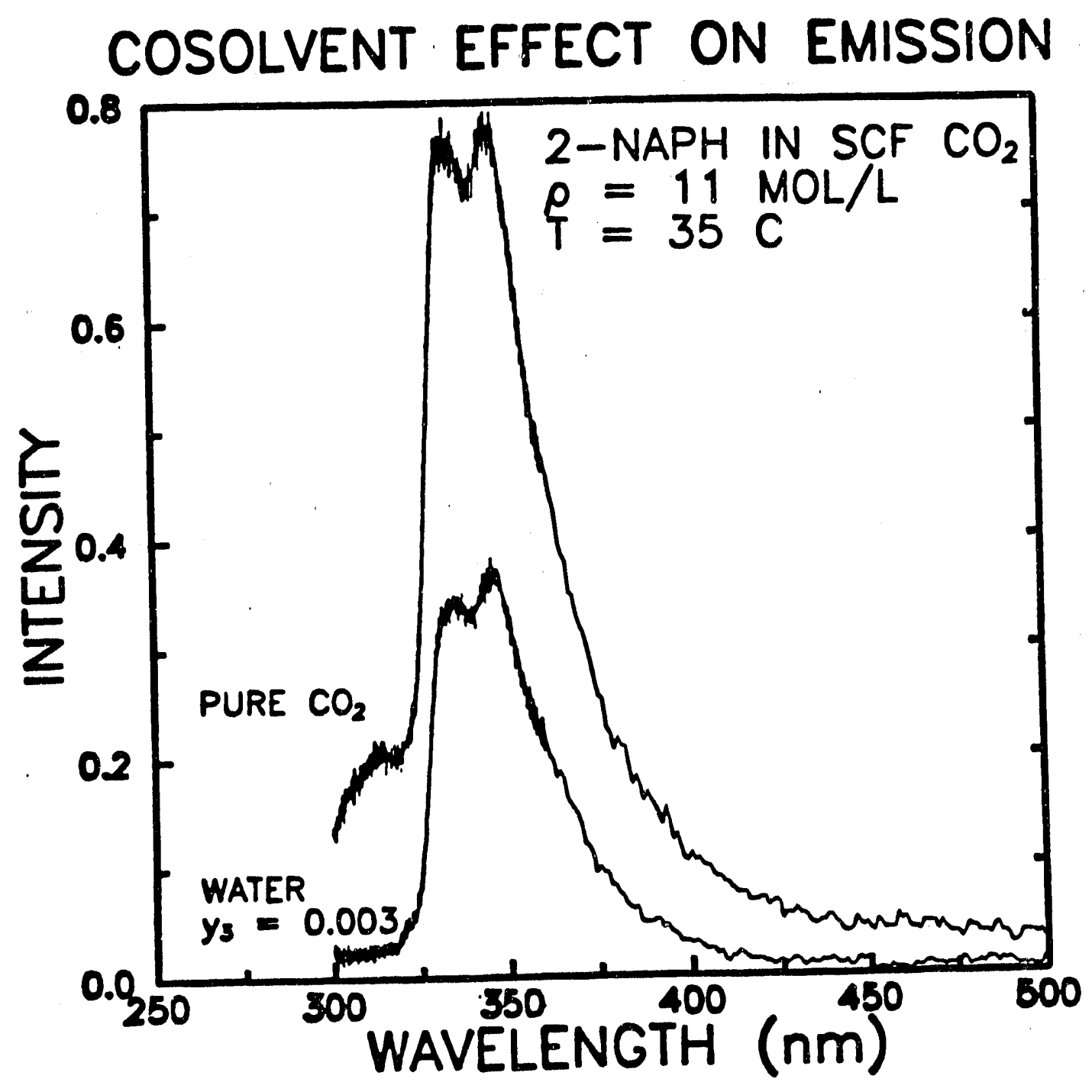

Figure 26. Effect of Water Cosolvent on the Fluorescence Emission of 2-Naphthol 


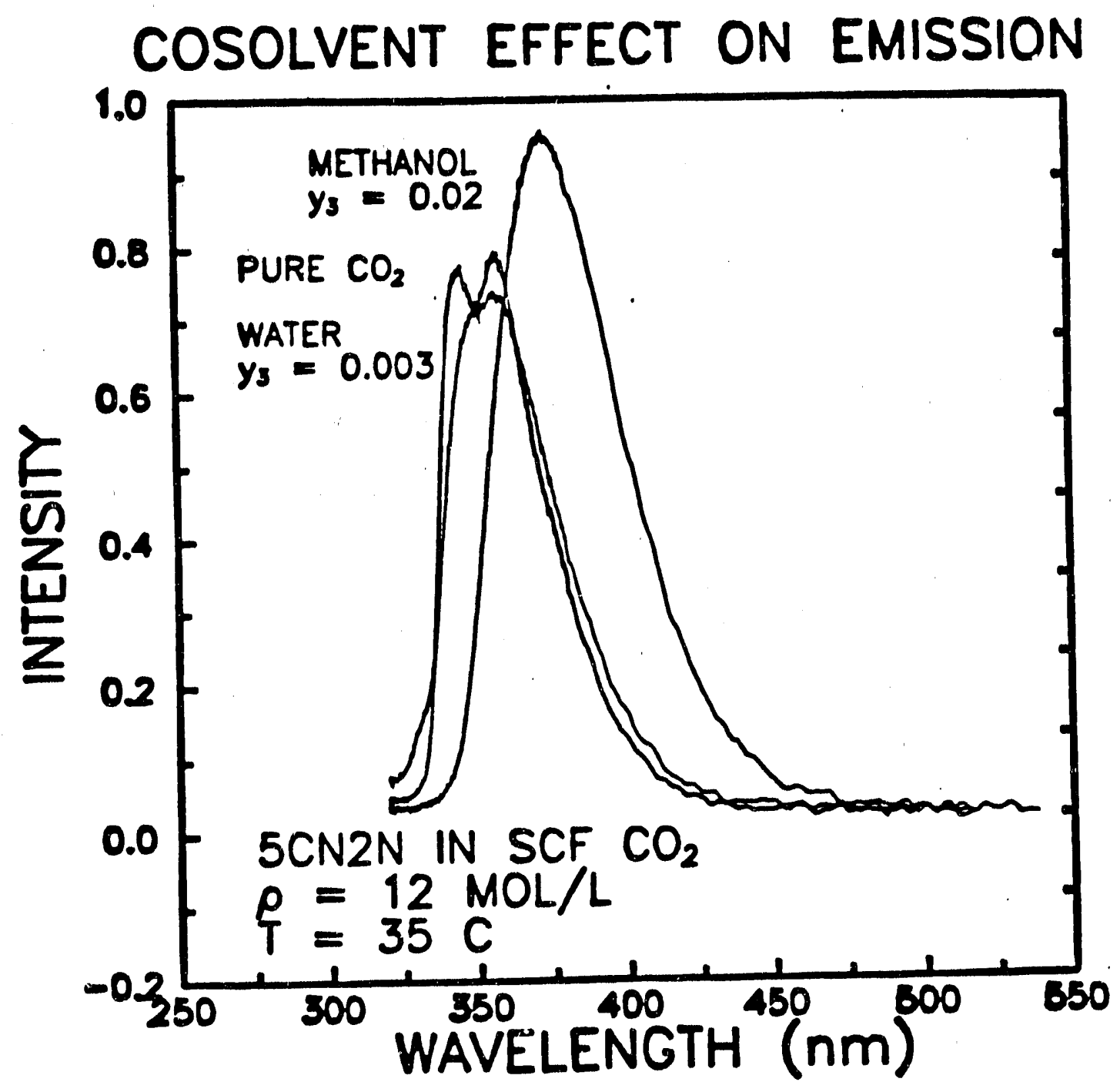

Figure 27. Effect of Water and Methanol Cosolvent on the Fluorescence Emission of 5-Cyano-2-Naphthol 


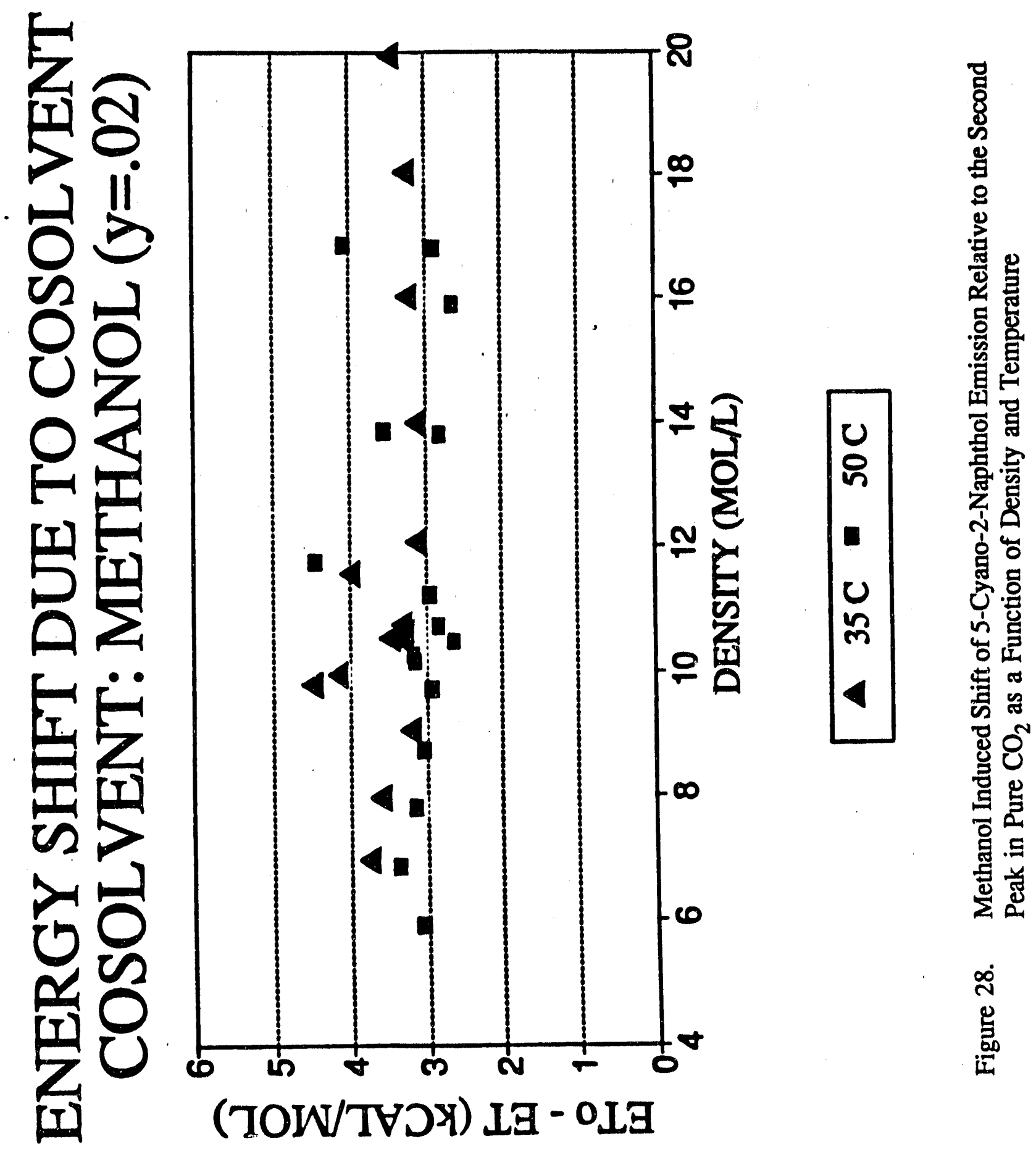




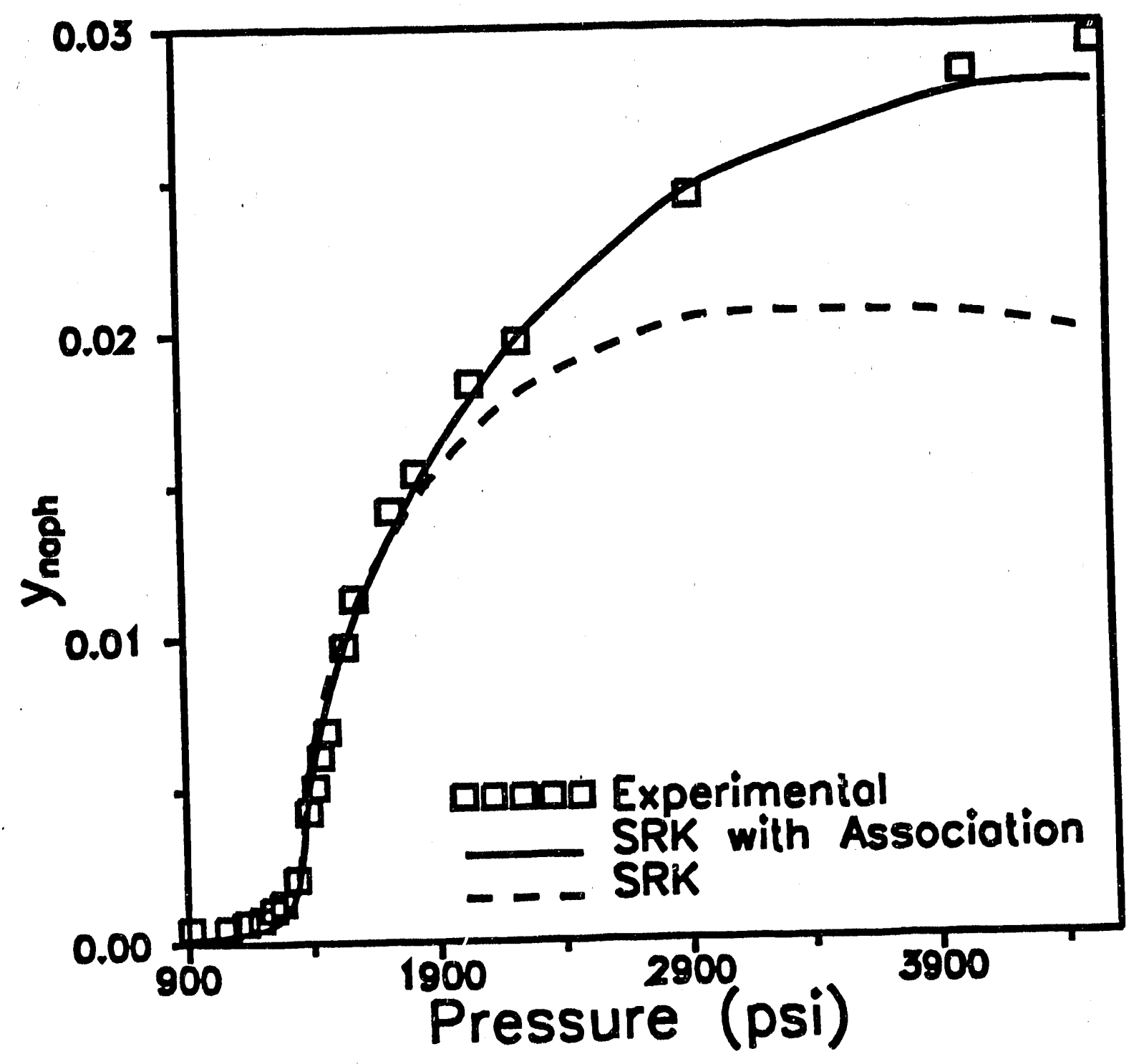

Figure 29. Solubility of naphthalene in $\mathrm{SCF} \mathrm{CO}_{2}$ at $45^{\circ} \mathrm{C}$; experimental and correlated. 


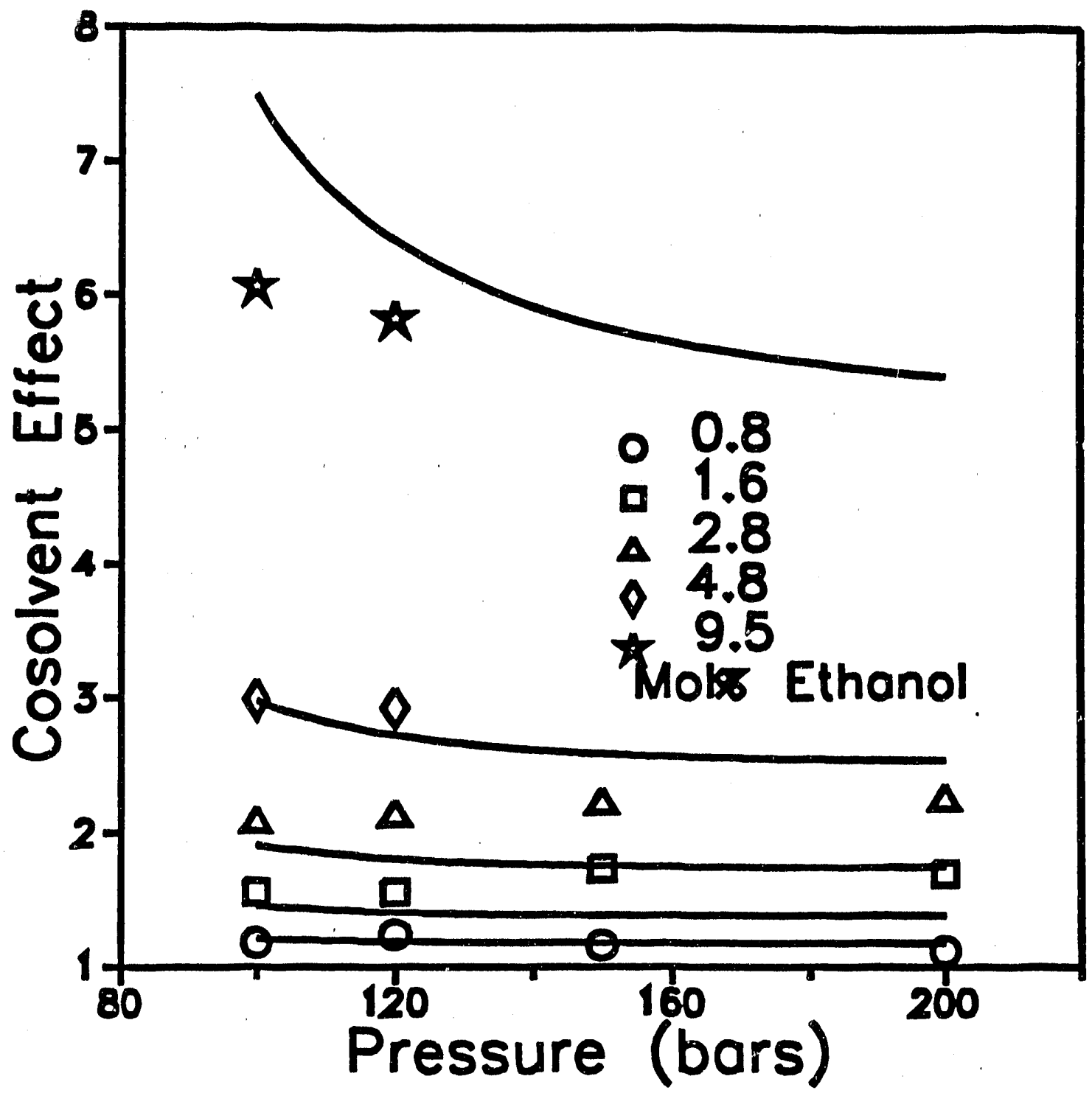

Figure 30. Cosolvent effect for carbazole in ethane/ethanol at $50^{\circ} \mathrm{C}$; experiment and model. 


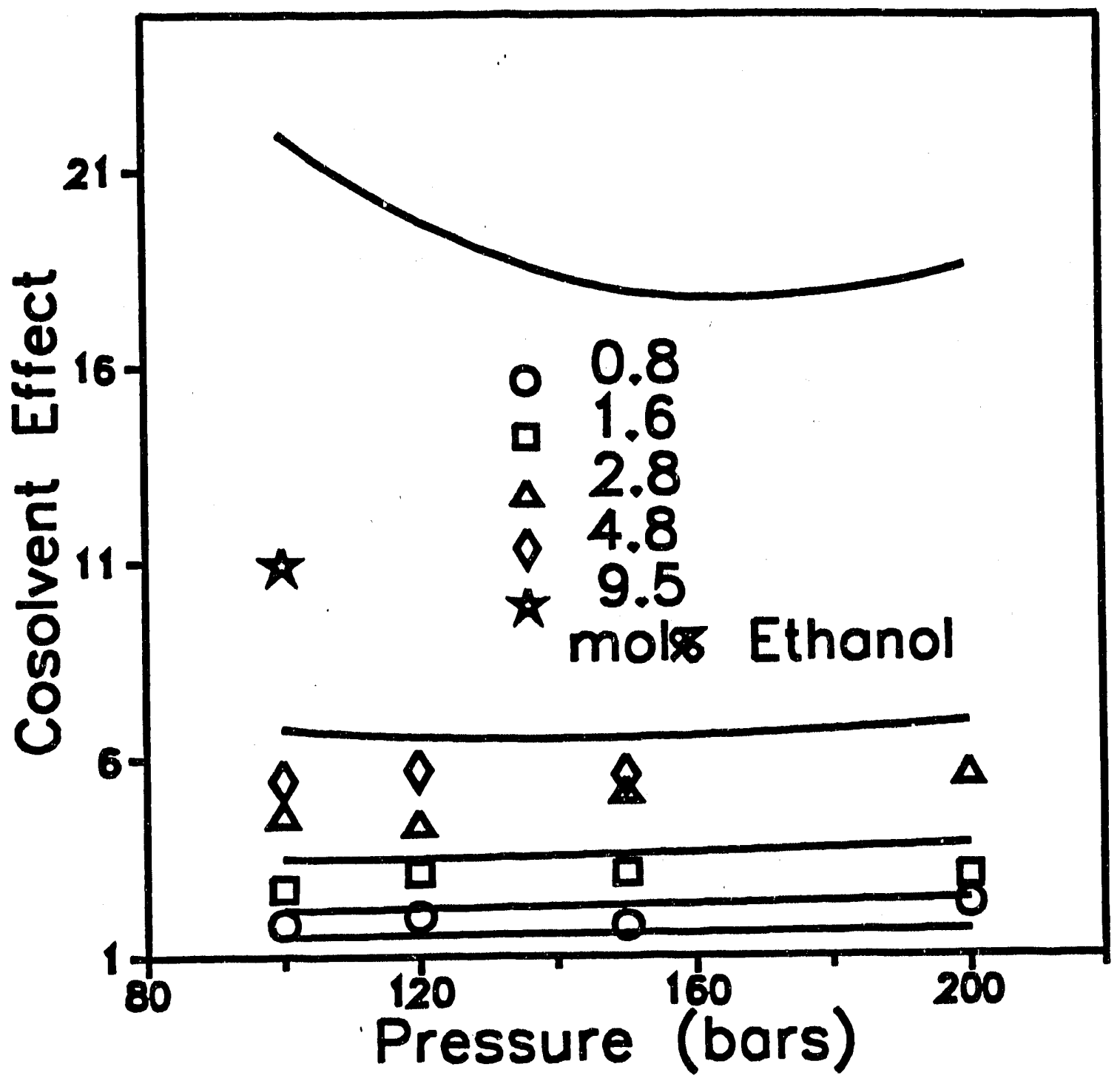

Figure 31. Cosolvent effect for 2-naphthol in ethane/ethanol at $50^{\circ} \mathrm{C}$; experiment and model. 

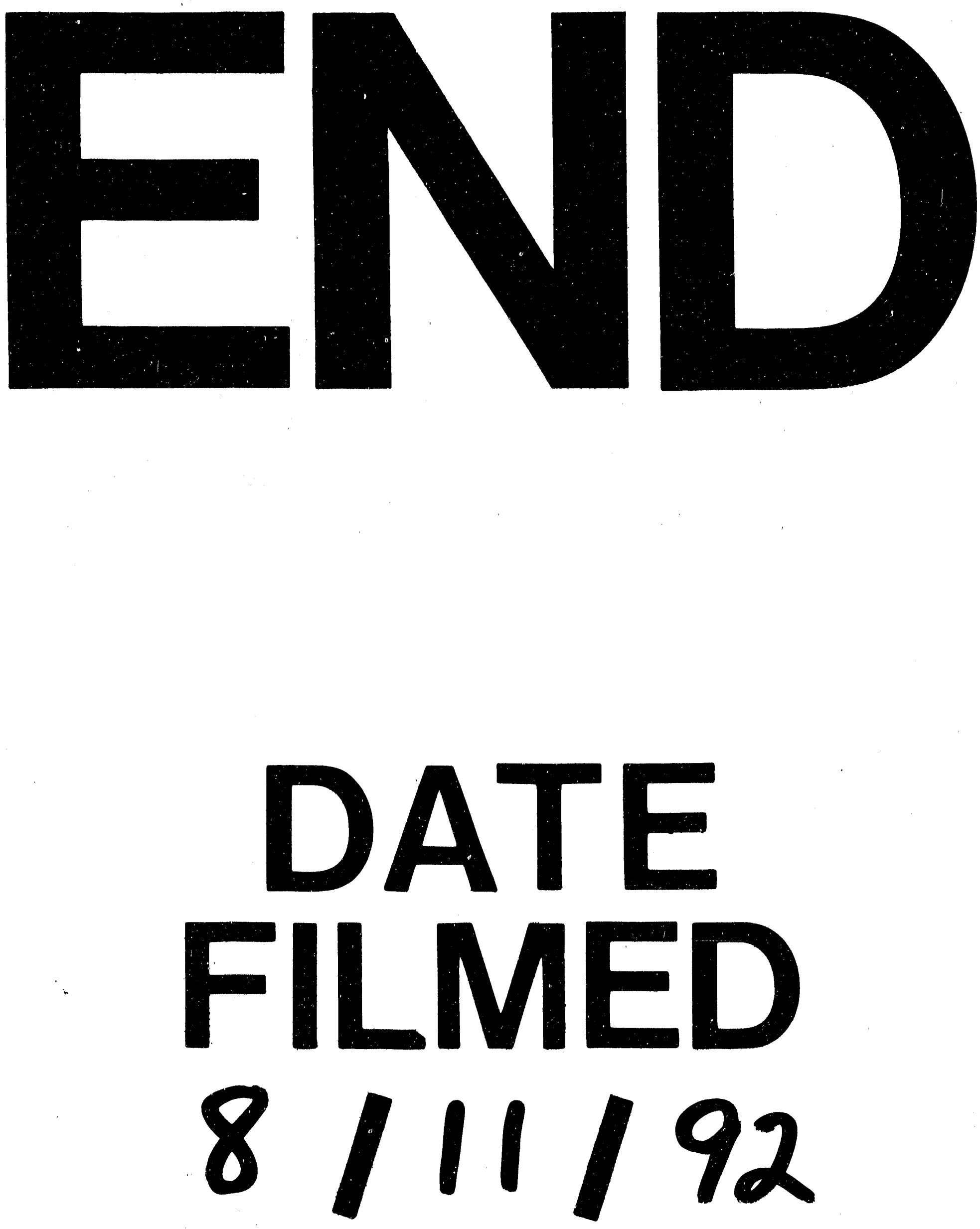

1 
